\title{
TITLE:
}

\section{The geometry and mechanics of generalized pseudo-rigid bodies}

$\operatorname{AUTHOR}(S)$ :

Iwai, Toshihiro

\section{CITATION:}

Iwai, Toshihiro. The geometry and mechanics of generalized pseudorigid bodies. Journal of Physics A: Mathematical and Theoretical 2010, 43(9): 95206.

\section{ISSUE DATE:}

2010-02-05

URL:

http://hdl.handle.net/2433/108457

\section{RIGHT:}

c Institute of Physics; This is not the published version. Please cite only the published version.; この論文は出版社版でありません。引用の際に は出版社版をご確認ご利用ください。 


\title{
The geometry and mechanics of generalized pseudo-rigid bodies
}

\author{
Toshihiro Iwai \\ Department of Applied Mathematics and Physics \\ Kyoto University, Kyoto 606-8501, Japan
}

The geometry and the mechanics of generalized pseudo-rigid bodies are studied. The configuration space of a generalized pseudo-rigid bodies is the linear group, $\mathcal{P}:=$ $\mathrm{GL}^{+}(n, \mathbf{R})$, of non-singular matrices with positive determinant. It admits the left and right actions of $\mathrm{SO}(n)$ and the two-sided action of $\mathrm{SO}(n) \times \mathrm{SO}(n)$. The left and right $\mathrm{SO}(n)$ actions on $\mathcal{P}$ are both free, so that $\mathcal{P}$ is made into respective principal fiber bundles according to the left and right $\mathrm{SO}(n)$ actions, and further left and right connections can be defined on the respective fiber bundles. However, the two-sided $\mathrm{SO}(n) \times \mathrm{SO}(n)$ action is not free on $\mathcal{P}$, and hence $\mathcal{P}$ is not made into a principal fiber bundle with respect to this action. In spite of this, if $\mathcal{P}$ is restricted to an open dense subset $\dot{\mathcal{P}}$, the isotropy subgroup at each point of $\dot{\mathcal{P}}$ is a finite discrete group, so that the quotient space $(\mathrm{SO}(n) \times \mathrm{SO}(n)) \backslash \dot{\mathcal{P}}$ becomes a manifold, and further one can define a connection on $\dot{\mathcal{P}}$, which will be called a bi-connection. The bi-connection is used to reduce the pseudo-rigid body system on $T^{*} \dot{\mathcal{P}}$ with the $\mathrm{SO}(n) \times \mathrm{SO}(n)$ symmetry. Though in the cotangent bundle reduction theorem and its variants, one usually assumes that the action of a Lie group on the configuration space is free, or that the isotropy subgroup of the Lie group is trivial, the reduction procedure works well if the isotropy subgroup is not trivial but a finite group. As an application of the reduction procedure, relative equilibria are discussed in relation with the reduced Hamilton and Lagrange equations of motion. A necessary and sufficient condition is given for a relative equilibrium in terms of an amended potential on the reduced phase space. 


\section{Introduction}

A pseudo-rigid body (or affine-rigid body) is a body which is deformable by orientation preserving linear maps [1]. The pseudo-rigid body can be viewed as a generalization of a rigid body and, at the same time, as a particular case of continuum. According to Dirichlet and Riemann, a Riemann ellipsoid is a self-gravitating, constant mass-density fluid with an ellipsoidal boundary and with a velocity field that is a linear function of the Cartesian position coordinates in an inertial center-of-mass frame $[3,4,5]$. The classical theory of Riemann ellipsoids was summarized and further developed in [6].

This article studies the geometry and mechanics of pseudo-rigid bodies. The configuration space of a generalized pseudo-rigid body is the linear group, $\mathrm{GL}^{+}(n, \mathbf{R})$, of non-singular matrices with positive determinant. The $\mathrm{GL}^{+}(n, \mathbf{R})$ may be viewed as the center-of-mass system of $n+1$ particles in $\mathbf{R}^{n}$, where the column vectors of $X \in \mathrm{GL}^{+}(n, \mathbf{R})$ are viewed as the Jacobi vectors for the $n+1$ particles which are assumed to form configurations such that $\operatorname{det} X>0$.

The $\mathrm{GL}^{+}(n, \mathbf{R})$ admits the left and right actions of $\mathrm{SO}(n)$. Since respective actions are free, $\mathrm{GL}^{+}(n, \mathbf{R})$ is made into a principal fiber bundle, and endowed with a connection in respective manners. The connections that Rosensteel defined on $\operatorname{GL}^{+}(3, \mathbf{R})$ [5] are examples of these connections. In contrast with this, one may consider the left and right $\mathrm{SO}(n)$ actions simultaneously. However, the two-sided $\mathrm{SO}(n) \times \mathrm{SO}(n)$ action on $\mathrm{GL}^{+}(n, \mathbf{R})$ is not free, so that $\mathrm{GL}^{+}(n, \mathbf{R})$ is not made into a principal bundle, and one cannot define a connection on $\mathrm{GL}^{+}(n, \mathbf{R})$ in the usual manner. In spite of this, if $\mathrm{GL}^{+}(n, \mathbf{R})$ is restricted to an open dense subset, the isotropy subgroup at each point of it is a finite discrete subgroup, and hence the orbit space by the two-sided action becomes a manifold, so that the idea of connection can work in this case. According to the decomposition of the tangent space into a direct sum of horizontal and vertical subspaces, a connection form can be defined, which will be called a bi-connection. In describing the connection form, the "bi-inertia" tensor is introduced. The introduction of the bi-inertia tensor and the bi-connection gives a new insight into the study of pseudo-rigid bodies or Riemann ellipsoids from the view point of symmetry reduction theory. If $\mathrm{GL}^{+}(n, \mathbf{R})$ is endowed with a natural Riemannian metric, the bi-connection is shown to be equal to a mechanical connection [7] associated with a locked inertia tensor with respect to $\mathrm{SO}(n) \times \mathrm{SO}(n)$ action. 
The mechanics for pseudo-rigid bodies is set up on the tangent/cotangent bundle over $\mathrm{GL}^{+}(n, \mathbf{R})$, and the pseudo-rigid body system with $\mathrm{SO}(n) \times \mathrm{SO}(n)$ symmetry is reduced by the use of the bi-connection. The reduction by the right $\mathrm{SO}(n)$ symmetry is also studied, which is a slight extension of $[4,8]$, though $\operatorname{Ref}[4,8]$ treated the mechanics in Poisson formalism.

If the group action of $\mathrm{SO}(n) \times \mathrm{SO}(n)$ were free, the reduction procedure to be performed for the pseudo-rigid body would be an example of the theorem known as the cotangent bundle reduction theorem [7, 9, 10]:

The cotangent bundle reduction theorem: Assume that $G$ acts freely and properly on a configuration space $Q$. Then the reduced symplectic manifold from $T^{*}(Q)$ is a fiber bundle over $T^{*}(Q / G)$ with fiber the coadjoint orbit $\mathcal{O}_{\mu}$ through $\mu \in \mathfrak{g}$.

However, since the isotropy subgroup of $\mathrm{SO}(n) \times \mathrm{SO}(n)$ is a finite group, the reduction procedure with the $\mathrm{SO}(n) \times \mathrm{SO}(n)$ symmetry will run in parallel with that in the above theorem with a slight modification.

The organization of this article is as follows: Section 2 contains geometric setting associated with the left and right $\mathrm{SO}(n)$ actions. The connections associated with the left and right $\mathrm{SO}(n)$ actions are defined, respectively. Section 3 deals with the reduction by the right $\mathrm{SO}(n)$ symmetry, which is a generalization of $[3,4]$. The reduction is performed in both Lagrangian and symplectic manners. In Sec. 4, Lagrangian and Hamiltonian mechanics are set up for pseudo-rigid bodies in terms of local coordinates associated with the $\mathrm{SO}(n) \times \mathrm{SO}(n)$ action on $\mathrm{GL}^{+}(n, \mathbf{R})$. Section 5 is specialized in the three-dimensional case. Section 6 is concerned with the $\mathrm{SO}(n) \times \mathrm{SO}(n)$ action. It starts with the study of the two-sided $\mathrm{SO}(n) \times \mathrm{SO}(n)$ action on the configuration space, and then gives the definitions of the bi-inertia tensor and the bi-connection. It is shown that the bi-inertia tensor is equal to the so-called locked inertia tensor [7] associated with the $\mathrm{SO}(n) \times \mathrm{SO}(n)$ action. Section 7 deals with the symplectic reduction of the pseudo-rigid body system with $\mathrm{SO}(n) \times \mathrm{SO}(n)$ symmetry by the effective use of the bi-connection. Further, the reduced system is described in terms of local coordinates. In Sec. 8, relative equilibria are discussed. A necessary and sufficient condition is given for an orbit of a one-parameter subgroup of $\mathrm{SO}(n) \times \mathrm{SO}(n)$ to be a solution curve of the pseudo-rigid body. Section 9 contains concluding remarks on reduction procedure and on the commuting reduction theorem $[11,12]$ in addition. 


\section{Geometric set-up with left and right $\mathrm{SO}(n)$ actions}

\subsection{Configuration space of a pseudo-rigid body}

The configuration space for a generalized pseudo-rigid body is

$$
\mathcal{P}:=\mathrm{GL}^{+}(n, \mathbf{R})=\left\{X \in \mathbf{R}^{n \times n} \mid \operatorname{det} X>0\right\},
$$

where $\mathbf{R}^{n \times n}$ denotes the set of $n \times n$ real matrices. The rotation group $\mathrm{SO}(n)$ acts freely on $\mathcal{P}$ both to the left and to the right,

$$
L_{g}: X \mapsto g X, \quad R_{h}: X \mapsto X h^{-1},
$$

where $g, h \in \mathrm{SO}(n)$. With respect to each $\mathrm{SO}(n)$ action, the configuration space can be made into a principal fiber bundle with the base space $\mathcal{Q}:=\operatorname{Sym}^{+}(n, \mathbf{R})$, the set of $n \times n$ positive definite real symmetric matrices. Then, according to the left and right $\mathrm{SO}(n)$ actions, the projection maps $\mathcal{P} \rightarrow \mathcal{Q}$ are given by

$$
\pi_{L}: X \mapsto X^{\top} X, \quad \pi_{R}: X \mapsto X X^{\top}
$$

respectively, where $X^{\top}$ denotes the transpose of $X$. These fiber bundles are trivial, since $\mathcal{Q}=\operatorname{Sym}^{+}(n, \mathbf{R})$ is contractible.

Though we can treat two-sided $\mathrm{SO}(n) \times \mathrm{SO}(n)$ action on $\mathcal{P}$ as well, we will postpone it to Sec. 6. However, we here use the two-sided action only to introduce a coordinate system in $\mathcal{P}=\mathrm{GL}^{+}(n, \mathbf{R})$. Let

$$
X=R A S^{-1}
$$

be a singular value decomposition of $X \in \mathcal{P}$, where $R, S \in \mathrm{SO}(n)$ and where $A$ is a diagonal matrix with positive real diagonal entries; $A=\operatorname{diag}\left(a_{1}, \cdots, a_{n}\right), a_{1} \geq$ $\cdots \geq a_{n}>0$. The decomposition is not unique. Another decomposition proves to be expressed as

$$
X=R D A D S^{-1}, \quad D=\operatorname{diag}\left(\varepsilon_{1}, \cdots, \varepsilon_{n}\right), \quad \varepsilon_{j}= \pm 1, \quad \operatorname{det} D=1 .
$$

Thus, we have the following. 
Lemma 2.1. Let $\dot{\mathcal{P}}$ and $\dot{\mathcal{D}}$ denote the subset of $\mathcal{P}$ whose singular values are all distinct and the set of diagonal matrices $A=\operatorname{diag}\left(a_{1}, \cdots, a_{n}\right)$ with entries such that $a_{1}>\cdots>$ $a_{n}>0$, respectively. Then the map

$$
\psi: \mathrm{SO}(n) \times \dot{\mathcal{D}} \times \mathrm{SO}(n) \rightarrow \dot{\mathcal{P}}, \quad(R, A, S) \mapsto X=R A S^{-1}
$$

is $2^{n-1}$-fold.

In spite of this, we are allowed to use $(R, A, S)$ as local coordinates of $\dot{\mathcal{P}}$ with $D$ fixed. In particular, $(R, A)$ and $(A, S)$ serve as local coordinates of $\dot{\mathcal{P}} / \mathrm{SO}(n)$ and $\mathrm{SO}(n) \backslash \dot{\mathcal{P}}$, respectively.

We denote the canonical inner product on $\mathbf{R}^{n \times n}$ by

$$
\langle A, B\rangle:=\operatorname{tr}\left(A^{\top} B\right), \quad A, B \in \mathbf{R}^{n \times n} .
$$

Restricted on the Lie algebra $\mathfrak{s o}(n)$ of $\mathrm{SO}(n)$, this inner product induces that on $\mathfrak{s o}(n)$. However, for $\mathfrak{s o}(n)$, we adopt the following definition and notation,

$$
\langle\xi, \eta\rangle_{\mathfrak{s o}(n)}=\frac{1}{2} \operatorname{tr}\left(\xi^{\top} \eta\right)=\sum_{i<j} \xi_{i j} \eta_{i j}, \quad \xi=\left(\xi_{i j}\right), \eta=\left(\eta_{i j}\right) \in \mathfrak{s o}(n) .
$$

With respect to this inner product, the dual space $\mathfrak{s o}(n)^{*}$ is identified with $\mathfrak{s o}(n)$.

The inner product (2.7) induces the Riemannian metric on $\mathcal{P}$;

$$
d s^{2}:=\operatorname{tr}\left(d X^{\top} d X\right)
$$

The reason why this metric is chosen is explained as follows: We assume that we have a pseudo-rigid body whose constituent particles are labeled by $\boldsymbol{u}_{\alpha}$ at the initial instance, where $\alpha$ may be continuous indices. Then, the kinetic energy $T$ is given by and expressed as

$$
2 T=\sum_{\alpha} \dot{X} \boldsymbol{u}_{\alpha} \cdot \dot{X} \boldsymbol{u}_{\alpha}=\operatorname{tr}\left(\dot{X}^{\top} \dot{X} \sum_{\alpha} \boldsymbol{u}_{\alpha} \boldsymbol{u}_{\alpha}^{\top}\right)
$$

where $\dot{X}$ and $\dot{X} \boldsymbol{u}_{\alpha}$ denote the time derivative of $X$ and the velocity vector of the constituent particle labeled by $\boldsymbol{u}_{\alpha}$, respectively, and where the center dot stands for the standard inner product on $\mathbf{R}^{n}$. If the $\alpha$ are continuous indices, the summation is replaced by the integral over the region occupied by the pseudo-rigid body at the 
initial instance. Since $Q:=\sum_{\alpha} \boldsymbol{u}_{\alpha} \boldsymbol{u}_{\alpha}^{\top}$ is a positive symmetric matrix for the pseudorigid body which has an open subset in it, there exists a positive symmetric matrix $Q^{1 / 2}$ such that the kinetic energy is written as $2 T=\operatorname{tr}\left(\left(\dot{X} Q^{1 / 2}\right)^{\top} \dot{X} Q^{1 / 2}\right)$. This allows us to choose $X Q^{1 / 2}$ to be a new variable in $\operatorname{GL}(n, \mathbf{R})$ and to rewrite $X Q^{1 / 2}$ as $X$. Then, the kinetic energy is put in the form $2 T=\operatorname{tr}\left(\dot{X}^{\top} \dot{X}\right)$, which leads to the metric $(2.9)$.

\subsection{Left and right connections}

We now define connections on the both fiber bundles $\pi_{L}: \mathcal{P} \rightarrow \mathrm{SO}(n) \backslash \mathcal{P}$ and $\pi_{R}$ : $\mathcal{P} \rightarrow \mathcal{P} / \mathrm{SO}(n)$.

Proposition 2.2. The maps $\mathcal{A}_{X}^{L}, \mathcal{A}_{X}^{R}: \mathfrak{s o}(n) \rightarrow \mathfrak{s o}(n)$ with $X \in \mathcal{P}$ are defined to be

$$
\mathcal{A}_{X}^{L}: \xi \mapsto X X^{\top} \xi+\xi X X^{\top}, \quad \mathcal{A}_{X}^{R}: \xi \mapsto X^{\top} X \xi+\xi X^{\top} X,
$$

and called the left and right inertia tensors, respectively. Both $\mathcal{A}_{X}^{L}$ and $\mathcal{A}_{X}^{R}$ are symmetric and positive-definite with respect to the inner product (2.8) on $\mathfrak{s o}(n)$, and then they are invertible. Furthermore, for $g, h \in \mathrm{SO}(n)$, the left and right inertia tensors transform, respectively, according to

$$
\begin{array}{ll}
\mathcal{A}_{g X}^{L}=\operatorname{Ad}_{g} \circ \mathcal{A}_{X}^{L} \circ \operatorname{Ad}_{g^{-1}}, & \mathcal{A}_{X h^{-1}}^{L}=\mathcal{A}_{X}^{L}, \\
\mathcal{A}_{g X}^{R}=\mathcal{A}_{X}^{R}, & \mathcal{A}_{X h^{-1}}^{R}=\operatorname{Ad}_{h} \circ \mathcal{A}_{X}^{R} \circ \operatorname{Ad}_{h^{-1}} .
\end{array}
$$

Since the proof is straightforward, we do not describe it here. Using the inertia tensors, we define connection forms.

Proposition 2.3. Let $\omega^{L}$ and $\omega^{R}$ be $\mathfrak{s o}(n)$-valued forms defined to be

$$
\begin{aligned}
& \omega_{X}^{L}:=\left(\mathcal{A}_{X}^{L}\right)^{-1}\left(d X X^{\top}-X d X^{\top}\right), \\
& \omega_{X}^{R}:=\left(\mathcal{A}_{X}^{R}\right)^{-1}\left(d X^{\top} X-X^{\top} d X\right),
\end{aligned}
$$

respectively, where $X \in \mathcal{P}$. Then, the $\omega^{L}$ and $\omega^{R}$ are shown to satisfy the following:

$$
\begin{gathered}
\omega_{X}^{L}(\xi X)=\xi, \quad \omega_{g X}^{L}=\operatorname{Ad}_{g} \circ \omega_{X}^{L}, \\
\omega_{X}^{R}\left(X \eta^{\top}\right)=\eta, \quad \omega_{X h^{-1}}^{R}=\operatorname{Ad}_{h} \circ \omega_{X}^{R},
\end{gathered}
$$

respectively, where $g, h \in \mathrm{SO}(n)$ and $\xi, \eta \in \mathfrak{s o}(n)$. The $\omega^{L}$ and $\omega^{R}$ are called the left and right connection forms on the fiber bundles $\pi_{L}: \mathcal{P} \rightarrow \mathcal{Q}$ and $\pi_{R}: \mathcal{P} \rightarrow \mathcal{Q}$, respectively. 
The proof is straightforward and omitted.

Let $\mathcal{O}_{X}^{L}$ and $\mathcal{O}_{X}^{R}$ denote the $\mathrm{SO}(n)$ orbits through $X \in \mathcal{P}$ by the left and right actions, respectively, and

$$
\begin{aligned}
& H_{X}^{L}:=\operatorname{ker} \omega_{X}^{L}, \quad V_{X}^{L}:=T_{X}\left(\mathcal{O}_{X}^{L}\right), \\
& H_{X}^{R}:=\operatorname{ker} \omega_{X}^{R}, \quad V_{X}^{R}:=T_{X}\left(\mathcal{O}_{X}^{R}\right),
\end{aligned}
$$

be the horizontal and vertical subspaces with respect to the connections $\omega^{L}$ and $\omega^{R}$, respectively. The horizontal subspaces turns out to be expressed as

$$
\begin{aligned}
& H_{X}^{L}=\left\{Y \in T_{X} \mathcal{P} \mid Y X^{\top}=X Y^{\top}\right\}, \\
& H_{X}^{R}=\left\{Y \in T_{X} \mathcal{P} \mid Y^{\top} X=X^{\top} Y\right\},
\end{aligned}
$$

respectively. As is well known, the tangent space at $X \in \mathcal{P}$ is decomposed into a direct sum of the vertical and horizontal subspaces with respect to $\omega^{L}$ and $\omega^{R}$, respectively,

$$
T_{X}(\mathcal{P})=H_{X}^{L} \oplus V_{X}^{L}, \quad T_{X}(\mathcal{P})=H_{X}^{R} \oplus V_{X}^{R}
$$

As is easily shown, the vertical and horizontal subspaces are orthogonal to each other with respect to the metric $(2.9)$, in both cases.

\subsection{Remarks on the left and right connections}

We now show that the connection $\omega^{L}$ is an extension of the connection that Guichardet defined for many-body systems [13]. For simplicity, we assume that we work in three dimensions. Let $\boldsymbol{x}_{\alpha}$ and $m_{\alpha}$ denote the positions and the mass of the particle labeled by $\alpha$, respectively. Then, the inertia tensor $A: \mathbf{R}^{3} \rightarrow \mathbf{R}^{3}$ is defined to be a linear map expressed as

$$
A(\boldsymbol{v})=\sum_{\alpha} m_{\alpha} \boldsymbol{x}_{\alpha} \times\left(\boldsymbol{v} \times \boldsymbol{x}_{\alpha}\right), \quad \boldsymbol{v} \in \mathbf{R}^{3},
$$

and the connection form $\omega$ is defined to be

$$
\omega=\mathcal{R}\left(\sum_{\alpha} m_{\alpha} \boldsymbol{x}_{\alpha} \times d \boldsymbol{x}_{\alpha}\right),
$$

where $\mathcal{R}: \mathbf{R}^{3} \rightarrow \mathfrak{s o}(3)$ is the isomorphism defined through $\mathcal{R}(\boldsymbol{a}) \boldsymbol{x}=\boldsymbol{a} \times \boldsymbol{x}$ with $\boldsymbol{a}, \boldsymbol{x} \in \mathbf{R}^{3}$ (see also [14]). Operated with $\mathcal{R}$, the defining equation of $A$ is put in the form

$$
\mathcal{R}(A(\boldsymbol{v}))=Q_{X} \mathcal{R}(\boldsymbol{v})+\mathcal{R}(\boldsymbol{v}) Q_{X}, \quad Q_{X}:=\sum_{\alpha} m_{\alpha} \boldsymbol{x}_{\alpha} \boldsymbol{x}_{\alpha}^{\top}
$$


In the case of a pseudo-rigid body, we may formally assume that the mass distribution is homogeneous and that $Q_{X}=X X^{\top}$. On rewriting $\mathcal{R}(\boldsymbol{v})$ as $\xi \in \mathfrak{s o}(3)$ and introducing $\mathcal{A}_{X}: \mathfrak{s o}(3) \rightarrow \mathfrak{s o}(3)$ by $\mathcal{A}_{X}(\mathcal{R}(\boldsymbol{v}))=\mathcal{R}(A(\boldsymbol{v}))$, we come to the definition of the left inertial tensor in three dimensions. Moreover, the connection form given above is brought into

$$
\omega=\mathcal{A}_{X}^{-1}\left(-\sum_{\alpha} m_{\alpha} \boldsymbol{x}_{\alpha} d \boldsymbol{x}_{\alpha}^{\top}+\sum_{\alpha} m_{\alpha} d \boldsymbol{x}_{\alpha} \boldsymbol{x}_{\alpha}^{\top}\right) .
$$

If $\sum_{\alpha} m_{\alpha} \boldsymbol{x}_{\alpha} d \boldsymbol{x}_{\alpha}^{\top}$ and $\sum_{\alpha} m_{\alpha} \boldsymbol{x}_{\alpha} d \boldsymbol{x}_{\alpha}^{\top}$ are replaced by $X d X^{\top}$ and $d X X^{\top}$, respectively, the above connection form fall into $\omega^{L}$ with $n=3$. Hence, the $\omega^{L}$ proves to be a natural generalization of Guichardet's connection.

For comparison's sake, we now show that the inertia tensors $\mathcal{A}_{X}^{L}$ and $\mathcal{A}_{X}^{R}$ are equal to the locked inertia tensors [7] associated with the left and right $\mathrm{SO}(n)$ actions, respectively. Let $\langle\langle\rangle$,$\rangle denote the inner product for vector fields on \mathcal{P}$, which is determined by (2.9). For $\xi \in \mathfrak{s o}(n)$, we denote the induced vector field on $\mathcal{P}$ by $\xi_{\mathcal{P}}^{L}(X)$ and $\xi_{\mathcal{P}}^{R}(X)$ according to the left and right actions, respectively. Then, the locked inertia tensors, $\mathbb{I}^{L}(X): \mathfrak{s o}(n) \rightarrow \mathfrak{s o}(n)^{*} \cong \mathfrak{s o}(n)$ and $\mathbb{I}^{R}(X): \mathfrak{s o}(n) \rightarrow \mathfrak{s o}(n)^{*} \cong \mathfrak{s o}(n)$ are defined accordingly by

$$
\left\langle\mathbb{I}^{L}(X) \xi, \eta\right\rangle_{\mathfrak{s o}(n)}=\left\langle\left\langle\xi_{\mathcal{P}}^{L}(X), \eta_{\mathcal{P}}^{L}(X)\right\rangle\right\rangle, \quad\left\langle\mathbb{I}^{R}(X) \xi, \eta\right\rangle_{\mathfrak{s o}(n)}=\left\langle\left\langle\xi_{\mathcal{P}}^{R}(X), \eta_{\mathcal{P}}^{R}(X)\right\rangle\right\rangle,
$$

respectively. Since $\xi_{\mathcal{P}}^{L}(X)=\xi X$ and $\xi_{\mathcal{P}}^{R}(X)=X \xi^{\top}$, we can calculate the right-hand sides of the above defining equations to obtain

$$
\left\langle\left\langle\xi_{\mathcal{P}}^{L}(X), \eta_{\mathcal{P}}^{L}(X)\right\rangle\right\rangle=\left\langle\mathcal{A}_{X}^{L}(\xi), \eta\right\rangle_{\mathfrak{s o}(n)}, \quad\left\langle\left\langle\xi_{\mathcal{P}}^{R}(X), \eta_{\mathcal{P}}^{R}(X)\right\rangle\right\rangle=\left\langle\mathcal{A}_{X}^{R}(\xi), \eta\right\rangle_{\mathfrak{s o}(n)} .
$$

Equations (2.27) and (2.28) are put together to imply that $\mathbb{I}^{L}(X)=\mathcal{A}_{X}^{L}$ and $\mathbb{I}^{R}(X)=$ $\mathcal{A}_{X}^{R}$. Needless to say, the left and right inertia tensors $\mathcal{A}_{X}^{L}$ and $\mathcal{A}_{X}^{R}$ are defined independently of the metric on $\mathcal{P}$. Further, the connections $\omega^{L}$ and $\omega^{R}$ are also viewed as mechanical connections [7]. As stated also in [7], the mechanical connection originated from $[13,15]$.

\subsection{Angular momentum and circulation}

We have here to remark that the connection forms are closely related with the angular momentum $L$ and the circulation $\Gamma$, which are defined to be

$$
L:=\dot{X} X^{\top}-X \dot{X}^{\top}, \quad \Gamma:=\dot{X}^{\top} X-X^{\top} \dot{X},
$$


respectively, where $\dot{X}$ denotes a tangent vector to $\mathcal{P}$ at $X \in \mathcal{P}$. The nomenclature "angular momentum" and "circulation" come from [4]. If $n=3, L$ and $\Gamma$ are associated with those vectors called the angular momentum and the circulation with respect to the space frame (see Sec. 5). From the definition of $L$ and $\Gamma$, one has

$$
\omega_{X}^{L}(\dot{X})=\left(\mathcal{A}_{X}^{L}\right)^{-1}(L), \quad \omega_{X}^{R}(\dot{X})=\left(\mathcal{A}_{X}^{R}\right)^{-1}(\Gamma)
$$

From (2.20), (2.21), and (2.30), it turns out that $\dot{X} \in H_{X}^{L} \Leftrightarrow L=0$ and $\dot{X} \in H_{X}^{R} \Leftrightarrow$ $\Gamma=0$. Put another way, a curve $X(t)$ is horizontal with respect to the left (resp. right) connection if and only if the angular momentum (resp. the circulation) vanishes along $X(t)$.

Further, we remark that the angular momentum and the circulation are, respectively, subject to the transformation, under the left and right $\mathrm{SO}(n)$ actions,

$$
\begin{array}{ll}
L(g X, g \dot{X})=\operatorname{Ad}_{g} L(X, \dot{X}), & L\left(X h^{-1}, \dot{X} h^{-1}\right)=L(X, \dot{X}), \\
\Gamma(g X, g \dot{X})=\Gamma(X, \dot{X}), & \Gamma\left(X h^{-1}, \dot{X} h^{-1}\right)=\operatorname{Ad}_{h} \Gamma(X, \dot{X}) .
\end{array}
$$

In terms of the local coordinates $(R, A, S)$, the angular momentum and the circulation are expressed, respectively, as

$$
\begin{aligned}
& L:=R\left(\Omega A^{2}+A^{2} \Omega-2 A \Lambda A\right) R^{-1}, \\
& \Gamma:=S\left(\Lambda A^{2}+A^{2} \Lambda-2 A \Omega A\right) S^{-1},
\end{aligned}
$$

where

$$
\Omega:=R^{-1} \dot{R}, \quad \Lambda:=S^{-1} \dot{S}
$$

\subsection{A remark on circulation}

Kelvin's circulation is defined to be the integral $\int_{C} \boldsymbol{v} \cdot d \boldsymbol{x}$, where $C$ is a closed loop in the fluid, and where $\boldsymbol{v}$ and $\boldsymbol{x}$ are the velocity field and the position vector of the fluid particle, respectively. According to the assumption of the Riemann ellipsoid, the position vector is linear in the labeling vector of the constituent particle; $\boldsymbol{x}=X \boldsymbol{u}$. Since the particle motion is expressed as $\boldsymbol{x}(t)=X(t) \boldsymbol{u}$, and since the loop $C$ is drawn at an arbitrarily fixed time, one has $\boldsymbol{v}=\dot{X} \boldsymbol{u}$ and $d \boldsymbol{x}=X d \boldsymbol{u}$. Pulling back $\boldsymbol{v}$ to the $\boldsymbol{u}$-space, one has $\boldsymbol{v} \cdot d \boldsymbol{x}=\boldsymbol{V} \cdot d \boldsymbol{u}$, where $\boldsymbol{V}=X^{\top} \dot{X} \boldsymbol{u}$. We denote by $C_{0}$ the closed curve 
pulled back from $C$ and set $V:=X^{\top} \dot{X}=\left(V_{i j}\right)$. Then, the circulation is rewritten, by the application of the Stokes theorem, as

$$
\int_{C} \boldsymbol{v} \cdot d \boldsymbol{x}=\int_{C_{0}} \boldsymbol{V} \cdot d \boldsymbol{u}=\int_{C_{0}} \sum_{i, j} V_{i j} u_{j} d u_{i}=\int_{S_{0}} d\left(\sum_{i, j} V_{i j} u_{j} d u_{i}\right),
$$

where $S_{0}$ is a surface with the boundary $C_{0}$. The right-hand side of the above equation is expanded to give

$$
\int_{S_{0}} d\left(\sum_{i, j} V_{i j} u_{j} d u_{i}\right)=\frac{1}{2} \int_{S_{0}} \sum_{i, j}\left(V_{j i}-V_{i j}\right) d u_{i} \wedge d u_{j}=\frac{1}{2} \operatorname{tr}\left(\left(V^{\top}-V\right)^{\top} \zeta\right),
$$

where $\zeta$ is the anti-symmetric matrix with entries

$$
\zeta_{i j}=\int_{S_{0}} d u_{i} \wedge d u_{j}
$$

The above discussion is true in $n$ dimensions, so that one has $\int_{C} \boldsymbol{v} \cdot d \boldsymbol{x}=\left\langle V^{\top}-V, \zeta\right\rangle_{\mathfrak{s o}(n)}$. Since the matrix $\zeta$ is arbitrary, we may refer to $V^{\top}-V=\dot{X}^{\top} X-X^{\top} \dot{X}$ as the circulation taking values in $\mathfrak{s o}(n)$. The exterior derivative of $\boldsymbol{v} \cdot d \boldsymbol{x}$ is called the volticity, and so is that of $\boldsymbol{V} \cdot d \boldsymbol{u}$. However, on account of the above equation, we have chosen to call $V^{\top}-V=\dot{X}^{\top} X-X^{\top} \dot{X}$ the circulation according to [4].

\section{Reduction by right $\mathrm{SO}(n)$ symmetry}

Suppose we have a Lagrangian $\mathcal{L}(X, \dot{X})$ on the tangent bundle $T(\mathcal{P})$. As is easily verified, the Euler-Lagrange equation is expressed as

$$
\frac{d}{d t}\left(\frac{\partial \mathcal{L}}{\partial \dot{X}}\right)-\frac{\partial \mathcal{L}}{\partial X}=0
$$

If the Lagrangian is right $\mathrm{SO}(n)$ invariant, i.e., $\mathcal{L}\left(X h^{-1}, \dot{X} h^{-1}\right)=\mathcal{L}(X, \dot{X})$ for $h \in$ $\mathrm{SO}(n)$, Noether's theorem provides the conserved quantity associated with a oneparameter subgroup $h(\tau)=\exp (\tau \eta)$ with $\tau \in \mathbf{R}$ and $\eta \in \mathfrak{s o}(n)$. Since the Lagrangian for a pseudo-rigid body is assumed to be of the form

$$
\mathcal{L}=\frac{1}{2} \operatorname{tr}\left(\dot{X}^{\top} \dot{X}\right)-\mathcal{U}(X)
$$

the conserved quantity takes the form

$$
\left\langle\frac{\partial \mathcal{L}}{\partial \dot{X}}, X \eta^{\top}\right\rangle=\left\langle\dot{X}^{\top} X-X^{\top} \dot{X}, \eta\right\rangle_{\mathfrak{s o}(n)}
$$


Since $\eta \in \mathfrak{s o}(n)$ is arbitrary, we find that the circulation $\Gamma=\dot{X}^{\top} X-X^{\top} \dot{X}$ is conserved. We note here that the Kelvin circulation theorem or the conservation of the circulation is known to be related to the particle relabeling symmetry $[16,17]$. In our case, the particle relabeling is associated with the right $\mathrm{SO}(n)$ action.

We wish to perform the reduction procedure in the Lagrangian formalism in order to show that the theory of Riemann ellipsoids $[3,4]$ is generalized to that in $n$ dimensions. For comparison's sake we apply also the (co)tangent bundle reduction theorem, since $\mathrm{SO}(n)$ acts freely and properly on $\mathcal{P}$ to the right.

\subsection{Lagrangian reduction}

Since the Lagrangian is right $\mathrm{SO}(n)$ invariant, the pseudo-rigid body can be reduced to that on $T(\mathcal{P}) / \mathrm{SO}(n)$. To derive reduced Euler-Lagrange equations on $T(\mathcal{P}) / \mathrm{SO}(n)$, we start by choosing adaptive coordinates on $T(\mathcal{P}) / \mathrm{SO}(n)$. Right $\mathrm{SO}(n)$ invariant quantities can serve as local coordinates on $T(\mathcal{P}) / \mathrm{SO}(n)$, among which we take up

$$
Q:=X X^{\top}, \quad N:=X \dot{X}^{\top} .
$$

The $Q$ and $N$ are called the quadrupole moment [2] and the shear tensor, respectively, and used in the study of Riemann ellipsoids [3, 4].

We here make remarks on the variables $Q$ and $N$. Let

$$
T(\mathcal{P}) \simeq \mathcal{P} \times \mathfrak{g l}(n, \mathbf{R})
$$

be the right trivialization of the tangent bundle of $\mathcal{P}=\mathrm{GL}^{+}(n, \mathbf{R})$. Then, the right and left actions of $\mathrm{SO}(n)$ are lifted to those on $\mathcal{P} \times \mathfrak{g l}(n, \mathbf{R})$ and expressed as

$$
\begin{aligned}
& (X, \eta) \mapsto\left(X h^{-1}, \eta\right), \quad(h, \eta) \in \mathrm{SO}(n) \times \mathfrak{g l}(n, \mathbf{R}), \\
& (X, \xi) \mapsto\left(g X, \operatorname{Ad}_{g} \xi\right), \quad(g, \xi) \in \mathrm{SO}(n) \times \mathfrak{g l}(n, \mathbf{R}),
\end{aligned}
$$

respectively. According to (3.6a), the factor space $T(\mathcal{P}) / \mathrm{SO}(n)$ turns out to be

$$
T(\mathcal{P}) / \mathrm{SO}(n) \simeq(\mathcal{P} / \mathrm{SO}(n)) \times \mathfrak{g l}(n, \mathbf{R})
$$

The variables $(Q, N)$ are viewed as coordinates of the right-hand side of the above equation. Furthermore, let $\mathfrak{g l}(n, \mathbf{R})$ be decomposed into

$$
\mathfrak{g l}(n, \mathbf{R})=\operatorname{Sym}(n, \mathbf{R}) \oplus \mathfrak{s o}(n),
$$


where $\operatorname{Sym}(n, \mathbf{R})$ denotes the linear space of $n \times n$ symmetric matrices. Then, Eq. (3.7) is brought into

$$
T(\mathcal{P}) / \mathrm{SO}(n) \simeq T(\mathcal{P} / \mathrm{SO}(n)) \oplus \widetilde{\mathfrak{s o}(n)},
$$

where the tangent bundle $T(\mathcal{P} / \mathrm{SO}(n))$ is identified with $\mathcal{P} / \mathrm{SO}(n) \times \operatorname{Sym}(n, \mathbf{R})$, since $\mathcal{P} / \mathrm{SO}(n) \simeq \operatorname{Sym}^{+}(n, \mathbf{R})$ is simply connected, and where $\widetilde{\mathfrak{s o}(n)}$ denotes the bundle defined through the right $\mathrm{SO}(n)$ action on $\mathcal{P} \times \mathfrak{s o}(n) ;(X, \xi) \mapsto\left(X h^{-1}, \mathrm{Ad}_{h} \xi\right)$ with $h \in \mathrm{SO}(n)$.

Now, we break $N$ into the symmetric and skew-symmetric parts to obtain

$$
N=\frac{1}{2}(-L+\dot{Q})
$$

where $\dot{Q}=\dot{X} X^{\top}+X \dot{X}^{\top}$ and where $L$ is the angular momentum given in (2.29). Then, the variables $(Q, \dot{Q})$ and $(Q, L)$ are regarded as coordinates of $T(\mathcal{P} / \operatorname{SO}(n))$ and $\widetilde{\mathfrak{s o}(n)}$, respectively. Under the left $\mathrm{SO}(n)$ action, they are subject to the transformation

$$
(Q, \dot{Q}) \mapsto\left(g Q g^{-1}, g \dot{Q} g^{-1}\right), \quad(Q, L) \mapsto\left(g Q g^{-1}, g L g^{-1}\right),
$$

respectively, where $g \in \mathrm{SO}(n)$.

We now derive Euler-Lagrange equations for a Lagrangian $\mathcal{L}(Q, N)$ which is invariant under the right $\mathrm{SO}(n)$ action, on the variational principle,

$$
\delta \int_{t_{0}}^{t_{1}} \mathcal{L}(Q, N) d t=0
$$

To begin with, we have to study infinitesimal variations of $Q$ and $N$. Through a straightforward calculation, we can relate $\delta Q$ and $\delta N$ to the right invariant infinitesimal variation $\delta X X^{-1}$;

$$
\begin{aligned}
& \delta Q=\delta X X^{-1} Q+Q\left(\delta X X^{-1}\right)^{\top} \\
& \delta N=\delta X X^{-1} N-\left(\delta X X^{-1} N\right)^{\top}+\frac{d}{d t}\left(Q\left(\delta X X^{-1}\right)^{\top}\right)
\end{aligned}
$$

By using these infinitesimal variation, the variations of the Lagrangian with respect to $Q$ and $N$ are put in the form

$$
\begin{aligned}
\left\langle\frac{\partial \mathcal{L}}{\partial Q}, \delta Q\right\rangle= & 2\left\langle\frac{\partial \mathcal{L}}{\partial Q} Q, \delta X X^{-1}\right\rangle \\
\left\langle\frac{\partial \mathcal{L}}{\partial N}, \delta N\right\rangle=\langle & \left.\left(\frac{\partial \mathcal{L}}{\partial N}-\left(\frac{\partial \mathcal{L}}{\partial N}\right)^{\top}\right) N^{\top}-\frac{d}{d t}\left(\frac{\partial \mathcal{L}}{\partial N}\right)^{\top} Q, \delta X X^{-1}\right\rangle \\
& +\frac{d}{d t}\left\langle\frac{\partial \mathcal{L}}{\partial N}, Q\left(\delta X X^{-1}\right)^{\top}\right\rangle
\end{aligned}
$$


respectively. On the variational principle together with the boundary condition $\delta X=0$ at $t=t_{0}$ and $t=t_{1}$, the variational integral with the integrand expressed as the sum of (3.15) and (3.16) provides the Euler-Lagrange equation:

Theorem 3.1. The Euler-Lagrange equation on $T(\mathcal{P}) / \mathrm{SO}(n)$ is given by

$$
\frac{d}{d t}\left(\frac{\partial \mathcal{L}}{\partial N}\right)^{\top}=\left(\frac{\partial \mathcal{L}}{\partial N}-\left(\frac{\partial \mathcal{L}}{\partial N}\right)^{\top}\right) N^{\top} Q^{-1}+2 \frac{\partial \mathcal{L}}{\partial Q}
$$

We now apply this equation to the Lagrangian of the form $\mathcal{L}=\mathcal{K}-\mathcal{U}$ with

$$
\mathcal{K}=\frac{1}{2} \operatorname{tr}\left(N^{\top} Q^{-1} N\right)=\frac{1}{2} \operatorname{tr}\left(\dot{X}^{\top} \dot{X}\right), \quad \mathcal{U}=\mathcal{U}(Q) .
$$

Since

$$
\frac{\partial \mathcal{K}}{\partial N}=Q^{-1} N, \quad \frac{\partial \mathcal{K}}{\partial Q}=-\frac{1}{2} Q^{-1} N N^{\top} Q^{-1},
$$

the Euler-Lagrange equation (3.17) are put in the form

$$
\begin{aligned}
& \frac{d N}{d t}=N^{\top} Q^{-1} N-2 Q \frac{\partial \mathcal{U}}{\partial Q}, \\
& \frac{d Q}{d t}=N^{\top}+N,
\end{aligned}
$$

where (3.20b) is a straightforward consequence of the definition of $Q$. If $n=3$, these equations, except for the pressure term, coincide with those obtained in [4] for the Riemann ellipsoid. We note also that the reduced equation (3.17) is put in the form different from that expected from the usual reduction theorem. We will perform the well-known reduction theorem in the next subsection for the sake of comparison.

\subsection{Symplectic reduction}

We now apply the symplectic reduction procedure along with the right $\mathrm{SO}(n)$ symmetry. Let $(P, X)$ be coordinates of $T^{*}(\mathcal{P})$. Then, the canonical one-form $\theta$ is defined to be

$$
\theta=\operatorname{tr}\left(P^{\top} d X\right)
$$

and the Hamiltonian associated with (3.2) is expressed as

$$
\mathcal{H}=\frac{1}{2} \operatorname{tr}\left(P^{\top} P\right)+\mathcal{U}
$$


which is invariant under the right $\mathrm{SO}(n)$ action. The momentum map associated with the right $\mathrm{SO}(n)$ symmetry is the circulation $\Gamma=P^{\top} X-X^{\top} P \in \mathfrak{s o}(n)^{*} \simeq \mathfrak{s o}(n)$. In fact, for an arbitrary infinitesimal transformation, $X \eta^{\top}$ with $\eta \in \mathfrak{s o}(n)$, associated with the right $\mathrm{SO}(n)$ action, we have

$$
\theta\left(X \eta^{\top}\right)=\left\langle P^{\top} X-X^{\top} P, \eta\right\rangle_{\mathfrak{s o}(n)}=\langle\Gamma, \eta\rangle_{\mathfrak{s o}(n)},
$$

where we have used the fact that $\operatorname{tr}\left(A^{\top} B\right)=0$ with $A$ and $B$ symmetric and antisymmetric matrices, respectively. Since the tangent bundle $T(\mathcal{P})$ and the cotangent bundle $T^{*}(\mathcal{P})$ is identified through the metric $(2.9), P \in T_{X}^{*}(\mathcal{P})$ is expressed as $P=$ $Y+X \eta^{\top}$, according to the (right) orthogonal decomposition (2.22), where $Y \in H_{X}^{R}$ and $\eta \in \mathfrak{s o}(n)$. The definition of $\Gamma$ and the decomposition of $P$ is put together to give $\Gamma=\mathcal{A}_{X}^{R}(\eta)$, so that one obtains $\eta=\left(\mathcal{A}_{X}^{R}\right)^{-1}(\Gamma)$. Thus, $P \in T_{X}^{*}(\mathcal{P})$ is put in the form

$$
P=Y+X\left(\left(\mathcal{A}_{X}^{R}\right)^{-1}(\Gamma)\right)^{\top}, \quad Y \in H_{X}^{R}
$$

The level manifold $\Gamma^{-1}(\gamma)$ with $\gamma \in \mathfrak{s o}(n)$ fixed is then expressed as

$$
\Gamma^{-1}(\gamma)=\left\{\left(X, Y+X\left(\left(\mathcal{A}_{X}^{R}\right)^{-1}(\gamma)\right)^{\top}\right) \mid X \in \mathcal{P}, Y \in H_{X}^{R}\right\}
$$

The isotropy subgroup $G_{\gamma}=\left\{h \in \mathrm{SO}(n) \mid \operatorname{Ad}_{h}(\gamma)=\gamma\right\}$ acts on $\Gamma^{-1}(\gamma)$ in the manner

$$
\left(X, Y+X\left(\left(\mathcal{A}_{X}^{R}\right)^{-1}(\gamma)\right)^{\top}\right) \mapsto\left(X h^{-1},\left(Y+X\left(\left(\mathcal{A}_{X}^{R}\right)^{-1}(\gamma)\right)^{\top}\right) h^{-1}\right) .
$$

Since $\left\{Y+X\left(\left(\mathcal{A}_{X}^{R}\right)^{-1}(\gamma)\right)^{\top}\right\} \simeq H_{X}^{R} \simeq T_{\pi_{R}(X)}^{*}(\mathcal{P} / \mathrm{SO}(n))$, and since $\mathrm{SO}(n) / G_{\gamma}$ is difffeomorphic with the (co)adjoint orbit $\mathcal{O}_{\gamma}$ through $\gamma$, the reduced phase space $\Gamma^{-1}(\gamma) / G_{\gamma}$ is expressed as

$$
\Gamma^{-1}(\gamma) / G_{\gamma} \simeq T^{*}(\mathcal{P} / \mathrm{SO}(n)) \times_{\mathcal{P} / \mathrm{SO}(n)} \widetilde{\mathcal{O}}_{\gamma}
$$

where $\widetilde{\mathcal{O}}_{\gamma}$ denotes the bundle over $\mathcal{P} / \mathrm{SO}(n)$ whose fiber is diffeomorphic with $\mathcal{O}_{\gamma}$.

We now calculate the reduced symplectic form on $\Gamma^{-1}(\gamma) / G_{\gamma}$. By using (3.24), we rewrite the canonical one-form $\theta$ as

$$
\begin{aligned}
\theta & =\frac{1}{2} \operatorname{tr}\left(\left(\mathcal{A}_{X}^{R}\right)^{-1}(\Gamma)\left(X^{\top} d X-d X^{\top} X\right)\right)+\operatorname{tr}\left(Y^{\top} d X\right) \\
& =\left\langle\left(\mathcal{A}_{X}^{R}\right)^{-1}(\Gamma), d X^{\top} X-X^{\top} d X\right\rangle_{\mathfrak{s o}(n)}+\operatorname{tr}\left(Y^{\top} d X\right) \\
& =\left\langle\Gamma, \omega_{X}^{R}\right\rangle_{\mathfrak{s o}(n)}+\langle Y, d X\rangle,
\end{aligned}
$$


where we have used the fact that $\mathcal{A}_{X}^{R}$ is a symmetric operator on $\mathfrak{s o}(n)$. Let $\iota_{\gamma}$ denote the inclusion map, $\iota_{\gamma}: \Gamma^{-1}(\gamma) \rightarrow T^{*}(\mathcal{P})$. Then, one has

$$
\iota_{\gamma}^{*} \theta=\left\langle\gamma, \omega_{X}^{R}\right\rangle_{\mathfrak{s o}(n)}+\langle Y, d X\rangle
$$

The second term of the right-hand side of the above equation projects to the canonical one-form on $T^{*}(\mathcal{P} / \mathrm{SO}(n))$.

The Hamiltonian (3.22) is put in the form

$$
\begin{aligned}
\mathcal{H} & =\frac{1}{2}\langle Y, Y\rangle+\frac{1}{2}\left\langle X\left(\left(\mathcal{A}_{X}^{R}\right)^{-1}(\Gamma)\right)^{\top}, X\left(\left(\mathcal{A}_{X}^{R}\right)^{-1}(\Gamma)\right)^{\top}\right\rangle+\mathcal{U} \\
& =\frac{1}{2}\langle Y, Y\rangle+\frac{1}{2}\left\langle\Gamma,\left(\mathcal{A}_{X}^{R}\right)^{-1}(\Gamma)\right\rangle_{\mathfrak{s o}(n)}+\mathcal{U}
\end{aligned}
$$

Then, we have

$$
\iota_{\gamma}^{*} \mathcal{H}=\frac{1}{2}\langle Y, Y\rangle+\frac{1}{2}\left\langle\gamma,\left(\mathcal{A}_{X}^{R}\right)^{-1}(\gamma)\right\rangle_{\mathfrak{s o}(n)}+\mathcal{U} .
$$

The first term of the right-hand side of the above equation projects to the kinetic energy on $T^{*}(\mathcal{P} / \mathrm{SO}(n))$.

Theorem 3.2. Let $\left(T^{*}(\mathcal{P}), d \theta, \mathcal{H}\right)$ be a Hamiltonian system for a pseudo-rigid body, where $\theta$ and $\mathcal{H}$ are given in (3.21) and (3.22), respectively, and where $\mathcal{U}$ is assumed to be invariant under the right $\mathrm{SO}(n)$ action. Then the system admits right $\mathrm{SO}(n)$ symmetry and have the circulation $\Gamma$ as the associated momentum map. For $\gamma \in$ $\mathfrak{s o}(n) \backslash\{0\}$, the reduced phase space $\Gamma^{-1}(\gamma) / G_{\gamma}$, where $G_{\gamma}$ denotes the isotropy subgroup at $\gamma$, is expressed as in (3.27), and the reduced symplectic form $\omega_{\gamma}$ and the reduced Hamiltonian $\mathcal{H}_{\gamma}$ are determined through $\iota_{\gamma}^{*} d \theta=\pi_{\gamma}^{*} \omega_{\gamma}$ and $\iota_{\gamma}^{*} \mathcal{H}=\pi_{\gamma}^{*} \mathcal{H}$, respectively, where $\iota_{\gamma}^{*} \theta$ and $\iota_{\gamma}^{*} \mathcal{H}$ are given in (3.29) and (3.31), respectively, and where $\pi_{\gamma}$ is the projection $\Gamma^{-1}(\gamma) \rightarrow \Gamma^{-1}(\gamma) / G_{\gamma}$.

The above reduction is a realization of the orbit bundle picture of the cotangent bundle reduction $[10]$ for $\mathcal{P}$.

\section{Mechanics for pseudo-rigid bodies}

In this section, we derive both the Euler-Lagrange and Hamilton equations on the variational principle in terms of local coordinates. 


\subsection{Lagrangian formalism}

We take $(R, A, S, \Omega, \dot{A}, \Lambda)$ as local coordinates on the tangent bundle $T(\mathcal{P})$ (see (2.33) for the definition of $\Omega$ and $\Lambda$ ). For a given Lagrangian $\mathcal{L}$ on $T(\mathcal{P})$, we derive EulerLagrange equations on the variational principle;

$$
\delta \int_{t_{0}}^{t_{1}} \mathcal{L}(R, A, S, \Omega, \dot{A}, \Lambda) d t=0,
$$

where the boundary conditions $\delta R=\delta S=0, \delta A=0$ are imposed at $t=t_{0}, t=t_{1}$. To carry out the variational calculation, we need the formulae on infinitesimal variations of $\Omega$ and $\Lambda$,

$$
\delta \Omega=\left[\Omega, R^{-1} \delta R\right]+\frac{d}{d t}\left(R^{-1} \delta R\right), \quad \delta \Lambda=\left[\Lambda, S^{-1} \delta S\right]+\frac{d}{d t}\left(S^{-1} \delta S\right),
$$

and further, the formula on the inner product on $\mathfrak{s o}(n)$,

$$
\langle\xi,[\eta, \zeta]\rangle_{\mathfrak{s o}(n)}=\langle[\xi, \eta], \zeta\rangle_{\mathfrak{s o}(n)}, \quad \xi, \eta, \zeta \in \mathfrak{s o}(n)
$$

On using these formulae, we can derive the Euler-Lagrange equations.

Theorem 4.1. For a Lagrangian $\mathcal{L}(R, A, S, \Omega, \dot{A}, \Lambda)$ on $T(\mathcal{P})$, the Euler-Lagrange equations are given by

$$
\begin{aligned}
& \frac{d}{d t} \frac{\partial \mathcal{L}}{\partial \dot{A}}=\frac{\partial \mathcal{L}}{\partial A}, \\
& \frac{d}{d t} \frac{\partial \mathcal{L}}{\partial \Omega}=R^{\top} \frac{\partial \mathcal{L}}{\partial R}-\left(\frac{\partial \mathcal{L}}{\partial R}\right)^{\top} R+\left[\frac{\partial \mathcal{L}}{\partial \Omega}, \Omega\right], \\
& \frac{d}{d t} \frac{\partial \mathcal{L}}{\partial \Lambda}=S^{\top} \frac{\partial \mathcal{L}}{\partial S}-\left(\frac{\partial \mathcal{L}}{\partial S}\right)^{\top} S+\left[\frac{\partial \mathcal{L}}{\partial \Lambda}, \Lambda\right] .
\end{aligned}
$$

We now work with the kinetic energy of the pseudo-rigid body, which is given in (3.2). After a straightforward calculation, we obtain the kinetic energy in the form

$$
\mathcal{K}=\frac{1}{2} \operatorname{tr}\left(\dot{X}^{\top} \dot{X}\right)=\frac{1}{2} \operatorname{tr}\left(\dot{A}^{2}-A^{2}\left(\Omega^{2}+\Lambda^{2}\right)+2 \Omega A \Lambda A\right) .
$$

As is seen from the expression, the kinetic energy is independent of $R$ and $S$. This is a necessary consequence of the fact that the kinetic energy is invariant under the left and right $\mathrm{SO}(n)$ actions. We now assume that the potential function is also invariant under the same action, so that it has the form, $\mathcal{U}=\mathcal{U}(A)$. Then our Lagrangian 
becomes $\mathrm{SO}(n) \times \mathrm{SO}(n)$ invariant. We now write out the Euler-Lagrange equations for this Lagrangian. To do so, we need a notation for matrix operation. For a matrix $B:=\left(b_{i j}\right) \in \mathbf{R}^{n \times n}, \mathfrak{D}(B)$ denotes the diagonal matrix which shares the same diagonal entries as $B$;

$$
\mathfrak{D}(B):=\operatorname{diag}\left(b_{11}, \ldots, b_{n n}\right) .
$$

We have to write out every term appearing in the equations of Theorem 4.1. A straightforward calculation provides the differential of $\mathcal{K}$ in the form

$$
d \mathcal{K}=\langle E, d A\rangle+\langle\dot{A}, d \dot{A}\rangle+\left\langle R^{-1} L R, d \Omega\right\rangle_{\mathfrak{s o}(n)}+\left\langle S^{-1} \Gamma S, d \Lambda\right\rangle_{\mathfrak{s o}(n)},
$$

where

$$
E:=\mathfrak{D}\left(-\left(\Omega^{2}+\Lambda^{2}\right) A+(\Omega A \Lambda+\Lambda A \Omega)\right),
$$

and where we have used the fact that $d A$ is a diagonal matrix. Equation (4.7) results in

$$
\begin{array}{ll}
\frac{\partial \mathcal{K}}{\partial \Omega}=R^{-1} L R, & \frac{\partial \mathcal{K}}{\partial \Lambda}=S^{-1} \Gamma S, \\
\frac{\partial \mathcal{K}}{\partial A}=E, & \frac{\partial \mathcal{K}}{\partial \dot{A}}=\dot{A} .
\end{array}
$$

Hence, Theorem 4.1 reduces to the following.

Proposition 4.2. For the Lagrangian having the kinematic energy (4.5) and the potential $\mathcal{U}=\mathcal{U}(A)$, the Euler-Lagrange equations are put in the form

$$
\begin{aligned}
\frac{d}{d t} \dot{A} & =E-\frac{\partial \mathcal{U}}{\partial A}, \\
\frac{d}{d t}\left(R^{-1} L R\right) & =\left[R^{-1} L R, \Omega\right], \\
\frac{d}{d t}\left(S^{-1} \Gamma S\right) & =\left[S^{-1} \Gamma S, \Lambda\right],
\end{aligned}
$$

where $E$ is given in (4.8).

This result is already known in [1] in three dimensions and in [19] as the EulerPoincaré equations for Riemann ellipsoids. Further, these equations can be viewed as the reduced Euler-Lagrange equations named in [20]. The reason for this will be stated in Sec. 9. We note also that Eqs. (4.11b) and (4.11c) are equivalent to the conservation of $L$ and $\Gamma$, respectively. It is of use further to rewrite the kinetic energy in terms of $L$ and $\Gamma$,

$$
\mathcal{K}=\frac{1}{2}\langle\dot{A}, \dot{A}\rangle+\frac{1}{2}\left(\left\langle\Omega, R^{-1} L R\right\rangle_{\mathfrak{s o}(n)}+\left\langle\Lambda, S^{-1} \Gamma S\right\rangle_{\mathfrak{s o}(n)}\right)
$$




\subsection{Hamiltonian formalism}

We now treat the pseudo-rigid body in the Hamiltonian formalism. We introduce the variables $p_{A}, p_{\Omega}$, and $p_{\Lambda}$, as usual, by

$$
p_{A}=\frac{\partial \mathcal{L}}{\partial \dot{A}}, \quad p_{\Omega}=\frac{\partial \mathcal{L}}{\partial \Omega}, \quad p_{\Lambda}=\frac{\partial \mathcal{L}}{\partial \Lambda},
$$

respectively. This means that we have taken $\left(R, A, S, p_{\Omega}, p_{A}, p_{\Lambda}\right)$ as local coordinates of $T^{*}(\mathcal{P})$. The Hamiltonian is then defined to be

$$
\mathcal{H}=\left\langle p_{\Omega}, \Omega\right\rangle_{\mathfrak{s o}(n)}+\left\langle p_{\Lambda}, \Lambda\right\rangle_{\mathfrak{s o}(n)}+\left\langle p_{A}, \dot{A}\right\rangle-\mathcal{L}
$$

If the kinetic energy is chosen as in (4.12), and if the Lagrangian is of the form $\mathcal{L}=$ $\mathcal{K}-\mathcal{U}$, a straightforward calculation along with (4.13) provides

$$
\begin{aligned}
& p_{\Omega}=A^{2} \Omega+\Omega A^{2}-2 A \Lambda A=R^{-1} L R, \\
& p_{\Lambda}=A^{2} \Lambda+\Lambda A^{2}-2 A \Omega A=S^{-1} \Gamma S,
\end{aligned}
$$

and the Hamiltonian takes the form

$$
\mathcal{H}=\frac{1}{2}\left\langle p_{A}, p_{A}\right\rangle+\frac{1}{2}\left\langle p_{\Omega}, \Omega\right\rangle_{\mathfrak{s o}(n)}+\frac{1}{2}\left\langle p_{\Lambda}, \Lambda\right\rangle_{\mathfrak{s o}(n)}+\mathcal{U},
$$

where $\Omega$ and $\Lambda$ in the above equation should be $\mathfrak{s o}(n)$-valued functions of $p_{\Omega}, p_{\Lambda}$, and $A$, which are determined through (4.15a) and (4.15b) (see (6.29)).

The Hamilton equations of motion are derived on the variational principle. As a result, we obtain

Theorem 4.3. For a Hamiltonian $\mathcal{H}\left(R, A, S, p_{\Omega}, p_{A}, p_{\Lambda}\right)$ on $T^{*}(\mathcal{P})$, the Hamilton equations of motion are given by

$$
\begin{aligned}
& \frac{d}{d t} p_{A}=-\frac{\partial \mathcal{H}}{\partial A}, \\
& \frac{d}{d t} p_{\Omega}=\left[p_{\Omega}, \Omega\right]-R^{\top} \frac{\partial \mathcal{H}}{\partial R}+\left(\frac{\partial \mathcal{H}}{\partial R}\right)^{\top} R, \\
& \frac{d}{d t} p_{\Lambda}=\left[p_{\Lambda}, \Lambda\right]-S^{\top} \frac{\partial \mathcal{H}}{\partial S}+\left(\frac{\partial \mathcal{H}}{\partial S}\right)^{\top} S, \\
& \dot{A}=\frac{\partial \mathcal{H}}{\partial p_{A}}, \quad \Omega=\frac{\partial \mathcal{H}}{\partial p_{\Omega}}, \quad \Lambda=\frac{\partial \mathcal{H}}{\partial p_{\Lambda}} .
\end{aligned}
$$


Proposition 4.4. For the Hamiltonian (4.16) with $\mathcal{U}=\mathcal{U}(A)$, the Hamilton equations of motion reduce to $(4.17 \mathrm{~d})$ and

$$
\frac{d}{d t} p_{A}=-\frac{\partial \mathcal{H}}{\partial A}, \quad \frac{d}{d t} p_{\Omega}=\left[p_{\Omega}, \Omega\right], \quad \frac{d}{d t} p_{\Lambda}=\left[p_{\Lambda}, \Lambda\right]
$$

As in the Lagrangian formalism, we can show that the second and the third equations of (4.18) are equivalent to the conservation of the angular momentum $L=$ $R p_{\Omega} R^{-1}$ and the circulation $\Gamma=S p_{\Lambda} S^{-1}$, respectively. If we put $L=\lambda$ and $\Gamma=\gamma$ with $\lambda, \gamma \in \mathfrak{s o}(n)$, one has $p_{\Omega}=\operatorname{Ad}_{R}^{-1}(\lambda)$ and $p_{\Lambda}=\operatorname{Ad}_{S}^{-1}(\gamma)$, so that the second and third equations of (4.18) may be interpreted as equations on the (co)adjoint orbits through $\lambda$ and $\gamma$, respectively. We will discuss the reduction of the pseudo-rigid body system with $\mathrm{SO}(n) \times \mathrm{SO}(n)$ symmetry in Sec. 7 .

\section{$5 \quad$ In three dimensions}

We now specialize in the Euler-Lagrange equations for $\mathcal{P}=\operatorname{GL}^{+}(3, \mathbf{R})$. By using the isomorphism $\mathcal{R}: \mathbf{R}^{3} \rightarrow \mathfrak{s o}(3)$, we introduce vectors $\boldsymbol{\omega}$ and $\boldsymbol{\lambda}$, and further $\boldsymbol{a}$ through

$$
\mathcal{R}(\boldsymbol{\omega})=\Omega, \quad \mathcal{R}(\boldsymbol{\lambda})=\Lambda, \quad \boldsymbol{a}=\left(a_{i}\right),
$$

respectively, where $\boldsymbol{a}=\left(a_{i}\right)$ comes from $A=\operatorname{diag}\left(a_{1}, a_{2}, a_{3}\right)$. We define also $\boldsymbol{m}$ and $\boldsymbol{c}$ through

$$
\mathcal{R}(\boldsymbol{m})=R^{-1} L R, \quad \mathcal{R}(\boldsymbol{c})=S^{-1} \Gamma S,
$$

respectively. The vectors $\boldsymbol{m}$ and $\boldsymbol{c}$ are nothing but the angular momentum and the circulation with respect to the body frame [3], respectively. In contrast with these vectors, the angular momentum and the circulation with respect to the space frame are given by $R \boldsymbol{m}$ and $S \boldsymbol{c}$, respectively. Now, calculating $R^{-1} L R=\Omega A^{2}+A^{2} \Omega-2 A \Lambda A$ and $S^{-1} \Gamma S=\Lambda A^{2}+A^{2} \Lambda-2 A \Omega A$ in an explicit manner results in

$$
\boldsymbol{m}=\left(\begin{array}{c}
\left(a_{2}^{2}+a_{3}^{2}\right) \omega_{1}-2 a_{2} a_{3} \lambda_{1} \\
\left(a_{3}^{2}+a_{1}^{2}\right) \omega_{2}-2 a_{3} a_{1} \lambda_{2} \\
\left(a_{1}^{2}+a_{2}^{2}\right) \omega_{3}-2 a_{1} a_{2} \lambda_{3}
\end{array}\right), \quad \boldsymbol{c}=\left(\begin{array}{c}
\left(a_{2}^{2}+a_{3}^{2}\right) \lambda_{1}-2 a_{2} a_{3} \omega_{1} \\
\left(a_{3}^{2}+a_{1}^{2}\right) \lambda_{2}-2 a_{3} a_{1} \omega_{2} \\
\left(a_{1}^{2}+a_{2}^{2}\right) \lambda_{3}-2 a_{1} a_{2} \omega_{3}
\end{array}\right)
$$

respectively. The vector corresponding to $E=\mathfrak{D}\left(-\left(\Omega^{2}+\Lambda^{2}\right) A+(\Omega A \Lambda+\Lambda A \Omega)\right)$ is written out as

$$
\boldsymbol{E}=\left(\begin{array}{l}
a_{1}\left(\omega_{2}^{2}+\omega_{3}^{2}+\lambda_{2}^{2}+\lambda_{3}^{2}\right)-2 a_{2} \omega_{3} \lambda_{3}-2 a_{3} \omega_{2} \lambda_{2} \\
a_{2}\left(\omega_{3}^{2}+\omega_{1}^{2}+\lambda_{3}^{2}+\lambda_{1}^{2}\right)-2 a_{3} \omega_{1} \lambda_{1}-2 a_{1} \omega_{3} \lambda_{3} \\
a_{3}\left(\omega_{1}^{2}+\omega_{2}^{2}+\lambda_{1}^{2}+\lambda_{2}^{2}\right)-2 a_{1} \omega_{2} \lambda_{2}-2 a_{2} \omega_{1} \lambda_{1}
\end{array}\right)
$$


Then, the Euler-Lagrange equations (4.11a), (4.11b), and (4.11c) are brought into

$$
\begin{aligned}
\ddot{\boldsymbol{a}} & =\boldsymbol{E}-\nabla U(\boldsymbol{a}), \\
\dot{\boldsymbol{m}} & =\boldsymbol{m} \times \boldsymbol{\omega}, \\
\dot{\boldsymbol{c}} & =\boldsymbol{c} \times \boldsymbol{\lambda},
\end{aligned}
$$

respectively. These are well-known equations in the liquid drop model of nuclei [3].

In a similar manner, we find that the kinetic energy (4.12) is expressed as

$$
\mathcal{K}=\frac{1}{2} \sum_{i} \dot{a}_{i}^{2}+\frac{1}{2} \sum_{i, j, k}\left(\left(a_{i}^{2}+a_{j}^{2}\right)\left(\omega_{k}^{2}+\lambda_{k}^{2}\right)-4 a_{i} a_{j} \omega_{k} \lambda_{k}\right),
$$

where the summation over $i, j, k$ is taken cyclically. It turns out also that

$$
\frac{\partial \mathcal{K}}{\partial \boldsymbol{\omega}}=\boldsymbol{m}, \quad \frac{\partial \mathcal{K}}{\partial \boldsymbol{\lambda}}=\boldsymbol{c}, \quad \frac{\partial \mathcal{K}}{\partial \boldsymbol{a}}=\boldsymbol{E} .
$$

\section{Geometric set-up with $\mathrm{SO}(n) \times \mathrm{SO}(n)$ action}

\subsection{Two-sided action of $\mathrm{SO}(n) \times \mathrm{SO}(n)$}

To study the $\mathrm{SO}(n) \times \mathrm{SO}(n)$ symmetry of the pseudo-rigid body, we start with the two-sided action of $G=\mathrm{SO}(n) \times \mathrm{SO}(n)$ on $\mathcal{P}$, which are defined by

$$
X \longmapsto g X h^{-1}, \quad(g, h) \in \mathrm{SO}(n) \times \mathrm{SO}(n)
$$

Let $X=R A S^{-1}$ be a singular value decomposition of $X$, where $R, S \in \mathrm{SO}(n)$ and $A$ is a diagonal matrix whose diagonal entries are singular values of $X$. Then, the isotropy subgroups $G_{A}$ at $A$ and $G_{X}$ at $X$ prove to be isomorphic to each other. We deal with $G_{A}$. Let $A=\operatorname{diag}\left(a_{1}, a_{2}, \cdots, a_{n}\right)$ with $a_{1} \geq a_{2} \geq \cdots \geq a_{n}>0$. $G_{A}$ 's are determined, depending on various types of the singular values, and hence there are as many orbit types as the types of singular values. According to orbit types, $\mathcal{P}$ is stratified into strata, among which, the principal stratum, denoted by $\dot{\mathcal{P}}$, is the subset consisting of $X$ whose singular values are all distinct. In what follows, we treat $\dot{\mathcal{P}}$ in particular for the reason that the isotropy subgroup $G_{X}$ at $X \in \dot{\mathcal{P}}$ is a finite group, as is shown below, and this fact makes it feasible to perform the reduction procedure by $\mathrm{SO}(n) \times \mathrm{SO}(n)$ with slight modification, as will be seen in Sec. 7. In Sec. 9, we will 
give the isotropy subgroups depending on the singular value types in the case of $n=3$ (see (9.2)). Let $X=R A S^{-1} \in \dot{\mathcal{P}}$, where $A=\operatorname{diag}\left(a_{1}, \cdots, a_{n}\right)$ with $a_{1}>\cdots>a_{n}>0$. Then, the condition $g A h^{-1}=A$ for $(g, h) \in \mathrm{SO}(n) \times \mathrm{SO}(n)$ is solved by

$$
G_{A}=\left\{(D, D) \mid D=\operatorname{diag}\left(\varepsilon_{1}, \varepsilon_{2}, \cdots, \varepsilon_{n}\right), \quad \varepsilon_{j}= \pm 1, \operatorname{det} D=1\right\}
$$

which is isomorphic with $\left(\mathbf{Z}_{2}\right)^{n-1}$, where $\mathbf{Z}_{2}=\{ \pm 1\}$. The orbit $\mathcal{O}_{A}$ through $A$ by the $G$ action is then diffeomorphic with $(\mathrm{SO}(n) \times \mathrm{SO}(n)) /\left(\mathbf{Z}_{2}\right)^{n-1}$, where $\left(\mathbf{Z}_{2}\right)^{n-1}$ acts on $G=\mathrm{SO}(n) \times \mathrm{SO}(n)$ by $(g, h) \mapsto(g D, h D)$. Though the $\mathrm{SO}(n) \times \mathrm{SO}(n)$ action on $\dot{\mathcal{P}}$ is not free, the factor space is a manifold, as is shown below.

Proposition 6.1. Let $G=\mathrm{SO}(n) \times \mathrm{SO}(n)$, and $\dot{\mathcal{P}}$ denote the principal stratum or the subset consisting of $X \in \mathcal{P}$ whose singular values are all distinct. Then one has

$$
G \backslash \dot{\mathcal{P}} \simeq \mathcal{C}:=\left\{\boldsymbol{x}=\left(x_{j}\right) \in \mathbf{R}^{n} \mid x_{1}>\cdots>x_{n}>0\right\}
$$

The projection $\dot{\mathcal{P}} \rightarrow G \backslash \dot{\mathcal{P}}$ is denoted by $\pi$.

proof. Let $\mu_{j}(X)$ denote the singular values of $X \in \dot{\mathcal{P}}$ with $\mu_{1}(X)>\cdots>\mu_{n}(X)$ and $\boldsymbol{\mu}(X)=\left(\mu_{j}(X)\right)$ the vector consisting of the singular values. Then, $\boldsymbol{\mu}$ defines a map $\pi: \dot{\mathcal{P}} \rightarrow \mathcal{C}$. Since for any $\boldsymbol{x}=\left(x_{j}\right) \in \mathcal{C}$, a matrix $A=\operatorname{diag}\left(x_{1}, \cdots, x_{n}\right)$ maps to $\boldsymbol{\mu}(A)=\boldsymbol{x}$, the $\boldsymbol{\mu}$ is surjective. We now assume that $\boldsymbol{\mu}\left(X_{1}\right)=\boldsymbol{\mu}\left(X_{2}\right)=\left(a_{j}\right) \in \mathcal{C}$. Then, there exist $\left(R_{i}, S_{i}\right) \in G, i=1,2$, such that $X_{1}=R_{1} A S_{1}^{-1}$ and $X_{2}=R_{2} A S_{2}^{-1}$ with $A=\operatorname{diag}\left(a_{1}, \cdots, a_{n}\right)$. This implies that $X_{2}=R_{2} R_{1}^{-1} X_{1}\left(S_{2} S_{1}^{-1}\right)^{-1}$, so that $X_{1}$ and $X_{2}$ are sitting in the same orbit of $G$. It then follows that $G \backslash \dot{\mathcal{P}} \simeq \mathcal{C}$, and then $\pi$ may be identified with $\boldsymbol{\mu}$.

We return to $\mathcal{P}$ for the time being. According to the $G$-action on $\mathcal{P}$, the tangent space $T_{X}(\mathcal{P})$ at $X \in \mathcal{P}$ is decomposed into

$$
T_{X}(\mathcal{P})=V_{X} \oplus H_{X}
$$

where $V_{X}$ and $H_{X}$ are the vertical and horizontal subspaces which are defined, respectively, by $V_{X}=T_{A}\left(\mathcal{O}_{X}\right)$, the tangent space to the orbit $\mathcal{O}_{X}$ through $X$ and by 
$H_{X}=V_{X}^{\perp}$, where the orthogonal complement is taken with respect to the canonical metric (2.9) on $\mathcal{P}$. These subspaces prove to be expressed as

$$
\begin{aligned}
V_{X} & =\left\{\xi X+X \eta^{\top} \mid(\xi, \eta) \in \mathfrak{s o}(n) \times \mathfrak{s o}(n)\right\}, \\
H_{X} & =\left\{Y \in T_{X}(\mathcal{P}) \mid Y X^{\top}=X Y^{\top}, X^{\top} Y=Y^{\top} X\right\},
\end{aligned}
$$

respectively. We remark that the dimensions of $V_{X}$ and $H_{X}$ depend on $X$ in general.

On account of the singular value decomposition $X=R A S^{-1}$, the vertical and horizontal subspaces $V_{X}$ and $H_{X}$ are isomorphic with $V_{A}$ and $H_{A}$, respectively. If we restrict ourselves to $\dot{\mathcal{P}}$, for $X=R A S^{-1} \in \dot{\mathcal{P}}$, the $H_{A}$ is easily found to be expressed as

$$
H_{A}=\left\{\operatorname{diag}\left(u_{1}, \cdots, u_{n}\right) \mid u_{j} \in \mathbf{R}\right\}
$$

so that $\operatorname{dim} H_{X}=\operatorname{dim} H_{A}=n$. The dimension of $V_{X}$ with $X \in \dot{\mathcal{P}}$ is then $\operatorname{dim} V_{X}=$ $n^{2}-n=2 \operatorname{dim} \mathfrak{s o}(n)$, which implies also that $\xi X+X \eta^{\top}=0$ if and only if $\xi=\eta=0$, though this fact can be verified in a straightforward manner.

From (6.7), we see that the local coordinates $(R, A, S, \Omega, \dot{A}, \Lambda)$ of $T(\dot{\mathcal{P}})$, which we have used in Sec. 4, are in keeping with the $\mathrm{SO}(n) \times \mathrm{SO}(n)$ action; $\dot{A}$ is attributed to the horizontal vector at $A$ and $(\Omega, \Lambda)$ to the vertical vectors $\left(\Omega A, A \Lambda^{\top}\right)$ at $A$.

\subsection{Bi-connection}

In accordance with the decomposition (6.4) with $X \in \dot{\mathcal{P}}$, we may define a connection form on $\dot{\mathcal{P}}$. We start with the definition of the "bi-inertia tensor". In what follows, we describe elements $\xi \oplus \eta \in \mathfrak{s o}(n) \oplus \mathfrak{s o}(n)$ in the form of column vector, like $\left(\begin{array}{l}\xi \\ \eta\end{array}\right)$, and the inner product on $\mathfrak{s o}(n) \oplus \mathfrak{s o}(n)$ is defined through

$$
\left\langle\left(\begin{array}{c}
\xi^{\prime} \\
\eta^{\prime}
\end{array}\right),\left(\begin{array}{c}
\xi \\
\eta
\end{array}\right)\right\rangle_{\mathfrak{s o}(n) \oplus \mathfrak{s o}(n)}=\left\langle\xi^{\prime}, \xi\right\rangle_{\mathfrak{s o}(n)}+\left\langle\eta^{\prime}, \eta\right\rangle_{\mathfrak{s o}(n)} .
$$

Proposition 6.2. The bi-inertia tensor is defined to be a map $\mathcal{B}_{X}: \mathfrak{s o}(n) \oplus \mathfrak{s o}(n) \rightarrow$ $\mathfrak{s o}(n) \oplus \mathfrak{s o}(n)$ given by

$$
\mathcal{B}_{X}:=\left(\begin{array}{cc}
\mathcal{A}_{X}^{L} & -2 \operatorname{Ad}_{X} \\
-2 \operatorname{Ad}_{X}^{\top} & \mathcal{A}_{X}^{R}
\end{array}\right):\left(\begin{array}{c}
\xi \\
\eta
\end{array}\right) \mapsto\left(\begin{array}{c}
\mathcal{A}_{X}^{L}(\xi)-2 \operatorname{Ad}_{X}(\eta) \\
-2 \operatorname{Ad}_{X}^{\top}(\xi)+\mathcal{A}_{X}^{R}(\eta)
\end{array}\right),
$$

where $\left(\begin{array}{l}\xi \\ \eta\end{array}\right) \in \mathfrak{s o}(n) \oplus \mathfrak{s o}(n)$, and where $\operatorname{Ad}_{X}$ and $\operatorname{Ad}_{X}^{\top}$ are defined on $\mathfrak{s o}(n)$ through

$$
\operatorname{Ad}_{X}(\xi)=X \xi X^{T}, \quad \operatorname{Ad}_{X}^{\top}(\xi)=X^{\top} \xi X
$$


respectively. Then, the $\mathcal{B}_{X}$ is a positive symmetric operator for $X \in \dot{\mathcal{P}}$. Thus, $\mathcal{B}_{X}^{-1}$ exists for $X \in \dot{\mathcal{P}}$. Furthermore, $\mathcal{B}_{X}$ transforms according to

$$
\mathcal{B}_{g X h^{-1}}=\operatorname{Ad}_{(g, h)} \circ \mathcal{B}_{X} \circ \operatorname{Ad}_{(g, h)}^{-1}, \quad(g, h) \in \mathrm{SO}(n) \times \mathrm{SO}(n),
$$

where $\operatorname{Ad}_{(g, h)}:=\operatorname{Ad}_{g} \oplus \operatorname{Ad}_{h}$.

proof. From the definitions of $\mathcal{A}_{X}^{L}, \mathcal{A}_{X}^{R}, \operatorname{Ad}_{X}$, and $\operatorname{Ad}_{X}^{\top}$, we can easily verify that

$$
\begin{aligned}
& \left\langle\left(\begin{array}{c}
\xi^{\prime} \\
\eta^{\prime}
\end{array}\right),\left(\begin{array}{cc}
\mathcal{A}_{X}^{L} & -2 \operatorname{Ad}_{X} \\
-2 \operatorname{Ad}_{X}^{\top} & \mathcal{A}_{X}^{R}
\end{array}\right)\left(\begin{array}{l}
\xi \\
\eta
\end{array}\right)\right\rangle_{\mathfrak{s o}(n) \oplus \mathfrak{s o}(n)} \\
= & \left\langle\left(\begin{array}{cc}
\mathcal{A}_{X}^{L} & -2 \operatorname{Ad}_{X} \\
-2 \operatorname{Ad}_{X}^{\top} & \mathcal{A}_{X}^{R}
\end{array}\right)\left(\begin{array}{c}
\xi^{\prime} \\
\eta^{\prime}
\end{array}\right),\left(\begin{array}{l}
\xi \\
\eta
\end{array}\right)\right\rangle_{\mathfrak{s o}(n) \oplus \mathfrak{s o}(n)},
\end{aligned}
$$

so that $\mathcal{B}_{X}$ is symmetric. To verify the positive definiteness of $\mathcal{B}_{X}$ for $X \in \dot{\mathcal{P}}$, we put $X$ in the singular value decomposition form, $X=R A S^{-1}$, and set

$$
\operatorname{Ad}_{R}^{-1}(\xi)=\xi^{\prime}=\left(\xi_{i j}^{\prime}\right), \quad \operatorname{Ad}_{S}^{-1}(\eta)=\eta^{\prime}=\left(\eta_{i j}^{\prime}\right)
$$

Then, a straightforward calculation provides

$$
\begin{aligned}
& \left\langle\left(\begin{array}{c}
\xi \\
\eta
\end{array}\right),\left(\begin{array}{cc}
\mathcal{A}_{X}^{L} & -2 \operatorname{Ad}_{X} \\
-2 \operatorname{Ad}_{X}^{\top} & \mathcal{A}_{X}^{R}
\end{array}\right)\left(\begin{array}{c}
\xi \\
\eta
\end{array}\right)\right\rangle_{\mathfrak{s o}(n) \oplus \mathfrak{s o}(n)} \\
= & \sum_{i<j}\left(a_{i}-a_{j}\right)^{2}\left(\xi_{i j}^{\prime}\right)^{2}+2 \sum_{i<j} a_{i} a_{j}\left(\xi_{i j}^{\prime}-\eta_{i j}^{\prime}\right)^{2}+\sum_{i<j}\left(a_{i}-a_{j}\right)^{2}\left(\eta_{i j}^{\prime}\right)^{2} \geq 0 .
\end{aligned}
$$

This implies that when $a_{i} \neq a_{j}$ with $i \neq j$, the above quadratic form vanishes if and only if $\xi^{\prime}=\eta^{\prime}=0$, or if and only if $\xi=\eta=0$. Thus, we have verified that $\mathcal{B}_{X}$ is positive definite, if $X \in \dot{\mathcal{P}}$.

The verification of (6.11) is straightforward;

$$
\begin{aligned}
& \left(\begin{array}{cc}
\mathcal{A}_{g X h^{-1}}^{L} & -2 \mathrm{Ad}_{g X h^{-1}} \\
-2 \operatorname{Ad}_{g X h^{-1}}^{\top} & \mathcal{A}_{g X h^{-1}}^{R}
\end{array}\right) \\
= & \left(\begin{array}{cc}
\operatorname{Ad}_{g} \mathcal{A}_{X}^{L} \operatorname{Ad}_{g}^{-1} & -2 \operatorname{Ad}_{g} \operatorname{Ad}_{X} \operatorname{Ad}_{h}^{-1} \\
-2 \operatorname{Ad}_{g} \operatorname{Ad}_{X}^{\top} \operatorname{Ad}_{h}^{-1} & \operatorname{Ad}_{h} \mathcal{A}_{X}^{R} \operatorname{Ad}_{h}^{-1}
\end{array}\right) \\
= & \left(\begin{array}{cc}
\operatorname{Ad}_{g} & 0 \\
0 & \operatorname{Ad}_{h}
\end{array}\right)\left(\begin{array}{cc}
\mathcal{A}_{X}^{L} & -2 \mathrm{Ad}_{X} \\
-2 \mathrm{Ad}_{X}^{\top} & \mathcal{A}_{X}^{R}
\end{array}\right)\left(\begin{array}{cc}
\operatorname{Ad}_{g}^{-1} & 0 \\
0 & \operatorname{Ad}_{h}^{-1}
\end{array}\right),
\end{aligned}
$$


where we have used the transformation property of $\mathcal{A}_{X}^{L}$ and $\mathcal{A}_{X}^{R}$ (see (2.12) and (2.13)) and that for $\operatorname{Ad}_{X}$ and $\operatorname{Ad}_{X}^{\top}$,

$$
\operatorname{Ad}_{g X h^{-1}}=\operatorname{Ad}_{g} \operatorname{Ad}_{X} \operatorname{Ad}_{h}^{-1}, \quad \operatorname{Ad}_{g X h^{-1}}^{\top}=\operatorname{Ad}_{h} \operatorname{Ad}_{X}^{\top} \operatorname{Ad}_{g}^{-1} .
$$

This ends the proof.

We are now in a position to define a connection form on $\dot{\mathcal{P}}$, which we will refer to as a bi-connection with respect to the $\mathrm{SO}(n) \times \mathrm{SO}(n)$ action. We denote by $(\xi \oplus \eta)_{\mathcal{P}}(X)$ the vector field on $\mathcal{P}$ induced by $\xi \oplus \eta \in \mathfrak{s o}(n) \oplus \mathfrak{s o}(n)$ (see (6.5));

$$
(\xi \oplus \eta)_{\mathcal{P}}(X)=\xi X+X \eta^{\top}
$$

Proposition 6.3. Let $\omega^{B}$ be an $\mathfrak{s o}(n) \oplus \mathfrak{s o}(n)$-valued one-form defined to be

$$
\omega_{X}^{B}=\left(\begin{array}{cc}
\mathcal{A}_{X}^{L} & -2 \mathrm{Ad}_{X} \\
-2 \mathrm{Ad}_{X}^{\top} & \mathcal{A}_{X}^{R}
\end{array}\right)^{-1}\left(\begin{array}{c}
d X X^{\top}-X d X^{\top} \\
d X^{\top} X-X^{\top} d X
\end{array}\right) .
$$

Then, the $\omega^{B}$ satisfies the following,

$$
\begin{aligned}
& \omega_{X}\left((\xi \oplus \eta)_{\mathcal{P}}(X)\right)=\xi \oplus \eta, \quad \xi \oplus \eta \in \mathfrak{s o}(n) \oplus \mathfrak{s o}(n), \\
& \omega_{g X h^{-1}}^{B}=\operatorname{Ad}_{(g, h)} \circ \omega_{X}^{B}, \quad(g, h) \in \mathrm{SO}(n) \times \operatorname{SO}(n),
\end{aligned}
$$

and is called the bi-connection form.

proof. For a vertical vector $\xi X+X \eta^{\top}$ at $X$, one has

$$
\begin{aligned}
& \omega_{X}^{B}\left(\xi X+X \eta^{\top}\right) \\
= & \left(\begin{array}{cc}
\mathcal{A}_{X}^{L} & -2 \mathrm{Ad}_{X} \\
-2 \mathrm{Ad}_{X}^{\top} & \mathcal{A}_{X}^{R}
\end{array}\right)^{-1}\left(\begin{array}{c}
\xi X X^{\top}+X X^{\top} \xi-2 X \eta X^{\top} \\
-2 X^{\top} \xi X+\eta X^{\top} X+X^{\top} X \eta
\end{array}\right) \\
= & \left(\begin{array}{cc}
\mathcal{A}_{X}^{L} & -2 \mathrm{Ad}_{X} \\
-2 \operatorname{Ad}_{X}^{\top} & \mathcal{A}_{X}^{R}
\end{array}\right)^{-1}\left(\begin{array}{cc}
\mathcal{A}_{X}^{L} & -2 \mathrm{Ad}_{X} \\
-2 \operatorname{Ad}_{X}^{\top} & \mathcal{A}_{X}^{R}
\end{array}\right)\left(\begin{array}{l}
\xi \\
\eta
\end{array}\right)=\left(\begin{array}{l}
\xi \\
\eta
\end{array}\right) .
\end{aligned}
$$

The transformation property of $\omega_{X}^{B}$ results from that for $\mathcal{B}_{X}$;

$$
\begin{aligned}
\omega_{g X h^{-1}}^{B} & =\mathcal{B}_{g X h^{-1}}^{-1}\left(\begin{array}{c}
d\left(g X h^{-1}\right)\left(g X h^{-1}\right)^{\top}-g X h^{-1} d\left(g X h^{-1}\right)^{\top} \\
d\left(g X h^{-1}\right)^{\top} g X h^{-1}-\left(g X h^{-1}\right)^{\top} d\left(g X h^{-1}\right)
\end{array}\right) \\
& =\operatorname{Ad}_{(g, h)} \mathcal{B}_{X}^{-1} \operatorname{Ad}_{(g, h)}^{-1}\left(\begin{array}{cc}
\operatorname{Ad}_{g} & 0 \\
0 & \operatorname{Ad}_{h}
\end{array}\right)\left(\begin{array}{c}
d X X^{\top}-X d X^{\top} \\
d X^{\top} X-X^{\top} d X
\end{array}\right) \\
& =\operatorname{Ad}_{(g, h)} \omega_{X}^{B} .
\end{aligned}
$$

This ends the proof. 
It is now easy to see that

$$
\operatorname{ker} \omega_{X}^{B}=\left\{Y \in T_{X}(\dot{\mathcal{P}}) \mid Y X^{\top}-X Y^{\top}=Y^{\top} X-X^{\top} Y=0\right\}=H_{X}
$$

where $H_{X}$ has been given in (6.6).

\subsection{A remark on the bi-connection}

With (6.17) in mind, we now show that the bi-inertia tensor is equal to the locked inertia tensor $\mathbb{I}(X)[7]$ with respect to the two-sided $\mathrm{SO}(n) \times \mathrm{SO}(n)$ action, which is determined through

$$
\left\langle\mathbb{I}(X)(\xi \oplus \eta), \xi^{\prime} \oplus \eta^{\prime}\right\rangle_{\mathfrak{s o}(n) \oplus \mathfrak{s o}(n)}=\left\langle\left\langle(\xi \oplus \eta)_{\mathcal{P}}(X),\left(\xi^{\prime} \oplus \eta^{\prime}\right)_{\mathcal{P}}(X)\right\rangle\right\rangle
$$

A straightforward calculation of the right-hand side of the above definition provides

$$
\begin{aligned}
& \left\langle\left\langle(\xi \oplus \eta)_{\mathcal{P}}(X),\left(\xi^{\prime} \oplus \eta^{\prime}\right)_{\mathcal{P}}(X)\right\rangle\right\rangle \\
= & \operatorname{tr}\left((\xi X+X \eta)^{\top}\left(\xi^{\prime} X+X \eta^{\prime}\right)\right) \\
= & \left\langle\mathcal{A}_{X}^{L}(\xi)-2 \operatorname{Ad}_{X}(\xi), \xi^{\prime}\right\rangle_{\mathfrak{s o}(n)}+\left\langle-2 \operatorname{Ad}_{X}^{\top}(\eta)+\mathcal{A}_{X}^{R}(\eta), \eta^{\prime}\right\rangle_{\mathfrak{s o}(n)} \\
= & \left\langle\mathcal{B}_{X}(\xi \oplus \eta), \xi^{\prime} \oplus \eta^{\prime}\right\rangle_{\mathfrak{s o}(n) \oplus \mathfrak{s o}(n)} .
\end{aligned}
$$

This proves our assertion. Hence, the bi-connection is identified with the mechanical connection [7] as well. We note that the bi-inertia tensor is defined independently of the Riemannian metric on $\mathcal{P}$.

\subsection{Local expressions}

We now express the bi-connection form in terms of local coordinates. A straightforward calculation with $X=R A S^{-1}$ provides

$$
\omega_{R A S^{-1}}^{B}=\operatorname{Ad}_{(R, S)} \omega_{A}^{B}, \quad \omega_{A}^{B}:=R^{-1} d R \oplus S^{-1} d S .
$$

This implies that the bi-connection is flat.

We turn to the Lagrangian and the Hamiltonian. From (2.32), we have

$$
\left(\begin{array}{c}
R^{-1} L R \\
S^{-1} \Gamma S
\end{array}\right)=\left(\begin{array}{cc}
\mathcal{A}_{A}^{L} & -2 \mathrm{Ad}_{A} \\
-2 \mathrm{Ad}_{A} & \mathcal{A}_{A}^{R}
\end{array}\right)\left(\begin{array}{c}
\Omega \\
\Lambda
\end{array}\right),
$$


so that $R^{-1} L R \oplus S^{-1} \Gamma S=\mathcal{B}_{A}(\Omega \oplus \Lambda)$. The kinetic energy (4.12) in the Lagrangian formalism is then expressed, in terms of the bi-inertia tensor, as

$$
\mathcal{K}=\frac{1}{2}\langle\dot{A}, \dot{A}\rangle+\frac{1}{2}\left\langle\Omega \oplus \Lambda, \mathcal{B}_{A}(\Omega \oplus \Lambda)\right\rangle_{\mathfrak{s o}(n) \oplus \mathfrak{s o}(n)} .
$$

We proceed to the Hamiltonian. We have introduced the conjugate variables $p_{A}, p_{\Omega}$, and $p_{\Lambda}$ in (4.13) and found the relations, (4.15a) and (4.15b), of them to $\Omega$ and $\Lambda$, which are put in the form, like (6.27),

$$
\left(\begin{array}{c}
p_{\Omega} \\
p_{\Lambda}
\end{array}\right)=\left(\begin{array}{cc}
\mathcal{A}_{A}^{L} & -2 \mathrm{Ad}_{A} \\
-2 \mathrm{Ad}_{A} & \mathcal{A}_{A}^{R}
\end{array}\right)\left(\begin{array}{c}
\Omega \\
\Lambda
\end{array}\right)=\left(\begin{array}{cc}
\operatorname{Ad}_{R}^{-1} & 0 \\
0 & \operatorname{Ad}_{S}^{-1}
\end{array}\right)\left(\begin{array}{c}
L \\
\Gamma
\end{array}\right) .
$$

Then, the Hamiltonian (4.16) is put in the form

$$
\mathcal{H}=\frac{1}{2}\left\langle p_{A}, p_{A}\right\rangle+\frac{1}{2}\left\langle p_{\Omega} \oplus p_{\Lambda}, \mathcal{B}_{A}^{-1}\left(p_{\Omega} \oplus p_{\Lambda}\right)\right\rangle_{\mathfrak{s o}(n) \oplus \mathfrak{s o}(n)}+\mathcal{U}(A) .
$$

\section{$7 \quad$ Reduction by $\mathrm{SO}(n) \times \mathrm{SO}(n)$ symmetry}

\section{$7.1 \quad \mathrm{SO}(n) \times \mathrm{SO}(n)$ symmetry}

The Hamiltonian system we consider is the same as in Sec. 3, but it is assumed to be invariant under the action of $G=\mathrm{SO}(n) \times \mathrm{SO}(n)$ in this section. The conserved quantity associated with this symmetry is given by

$$
\theta\left(\xi X+X \eta^{\top}\right)=\left\langle P X^{\top}-X P^{\top}, \xi\right\rangle_{\mathfrak{s o}(n)}+\left\langle P^{\top} X-X^{\top} P, \eta\right\rangle_{\mathfrak{s o}(n)}
$$

From this, it turns out that the associated momentum map $J: T^{*}(\mathcal{P}) \rightarrow \mathfrak{s o}(n) \oplus \mathfrak{s o}(n)$ is expressed as

$$
J(X, P)=\left(P X^{\top}-X P^{\top}\right) \oplus\left(P^{\top} X-X^{\top} P\right) .
$$

Like (2.29), we denote the components of $J$ by

$$
L=P X^{\top}-X P^{\top}, \quad \Gamma=P^{\top} X-X^{\top} P,
$$

which are called also the angular momentum and the circulation, respectively. As is easily seen from (2.31a) and (2.31b), $J$ is adjoint-equivariant;

$$
J\left(g X h^{-1}, g P h^{-1}\right)=\operatorname{Ad}_{(g, h)} J(X, P), \quad(g, h) \in \mathrm{SO}(n) \times \mathrm{SO}(n) .
$$




\subsection{Symplectic reduction}

We study the level manifold $J^{-1}(\lambda \oplus \gamma)$ for a given $\lambda \oplus \gamma \in \mathfrak{s o}(n) \oplus \mathfrak{s o}(n)$. Our problem is to solve the equation $J(X, P)=\lambda \oplus \gamma$. Since $T^{*}(\mathcal{P}) \simeq T(\mathcal{P})$, we may put $P \in T_{X}^{*}(\mathcal{P})$ in the form $P=\xi X+Y+X \eta^{\top}$, where $\xi \oplus \eta \in \mathfrak{s o}(n) \oplus \mathfrak{s o}(n)$ and $Y \in H_{X}$. Plugging this into the equation $J(X, P)=\lambda \oplus \gamma$, we obtain

$$
\begin{array}{r}
\mathcal{A}_{X}^{L}(\xi)-2 \operatorname{Ad}_{X}(\eta)=\lambda, \\
-2 \operatorname{Ad}_{X}^{\top}(\xi)+\mathcal{A}_{X}^{R}(\eta)=\gamma,
\end{array}
$$

where $\operatorname{Ad}_{X}$ and $\operatorname{Ad}_{X}^{\top}$ are given in (6.10). The above equations are put together to be expressed as $\mathcal{B}_{X}(\xi \oplus \eta)=\lambda \oplus \gamma$ on account of (6.9). Since $\mathcal{B}_{X}$ is invertible if $X \in \dot{\mathcal{P}}$, we obtain $\xi \oplus \eta=\mathcal{B}_{X}^{-1}(\lambda \oplus \gamma)$ for $X \in \dot{\mathcal{P}}$. To get an explicit expression of $\xi \oplus \eta$, we now put $X$ in the singular value decomposition form, $X=R A S^{-1}$, and rewrite the above equations as

$$
\begin{aligned}
& \mathcal{A}_{A}^{L}\left(\xi^{\prime}\right)-2 \operatorname{Ad}_{A}\left(\eta^{\prime}\right)=\lambda^{\prime}, \\
& \mathcal{A}_{A}^{R}\left(\eta^{\prime}\right)-2 \operatorname{Ad}_{A}\left(\xi^{\prime}\right)=\gamma^{\prime},
\end{aligned}
$$

where

$$
\xi^{\prime}=\operatorname{Ad}_{R}^{-1}(\xi), \quad \eta^{\prime}=\operatorname{Ad}_{S}^{-1}(\eta), \quad \lambda^{\prime}=\operatorname{Ad}_{R}^{-1}(\lambda), \quad \gamma^{\prime}=\operatorname{Ad}_{S}^{-1}(\gamma)
$$

Now, these equations are easily solved for $\xi^{\prime}$ and $\eta^{\prime}$. Let

$$
\xi^{\prime}=\left(\xi_{i j}^{\prime}\right), \eta^{\prime}=\left(\eta_{i j}^{\prime}\right), \lambda^{\prime}=\left(\lambda_{i j}^{\prime}\right), \gamma^{\prime}=\left(\gamma_{i j}^{\prime}\right), \quad A=\operatorname{diag}\left(a_{1}, \cdots, a_{n}\right)
$$

Then, a straightforward calculation provides the solutions,

$$
\begin{aligned}
\xi_{i j}^{\prime} & =\frac{\lambda_{i j}^{\prime}\left(a_{i}^{2}+a_{j}^{2}\right)+2 a_{i} a_{j} \gamma_{i j}^{\prime}}{\left(a_{i}^{2}-a_{j}^{2}\right)^{2}}, \\
\eta_{i j}^{\prime} & =\frac{\gamma_{i j}^{\prime}\left(a_{i}^{2}+a_{j}^{2}\right)+2 a_{i} a_{j} \lambda_{i j}^{\prime}}{\left(a_{i}^{2}-a_{j}^{2}\right)^{2}} .
\end{aligned}
$$

Here, we note that $a_{i} \neq a_{j}$ with $i \neq j$ for $X \in \dot{\mathcal{P}}$. Using these $\xi^{\prime}$ and $\eta^{\prime}$, one obtains $\xi \oplus \eta=\left(\operatorname{Ad}_{R} \oplus \operatorname{Ad}_{S}\right)\left(\xi^{\prime} \oplus \eta^{\prime}\right)$. The level manifold is now expressed as

$$
\left.J^{-1}(\lambda \oplus \gamma)\right|_{T^{*}(\dot{\mathcal{P}})}=\left\{\left(X,\left(\mathcal{B}_{X}^{-1}(\lambda \oplus \gamma)\right)_{\mathcal{P}}(X)+Y\right) \mid X \in \dot{\mathcal{P}}, Y \in H_{X}\right\}
$$


where the notation (6.17) has been used for $\xi \oplus \eta=\mathcal{B}_{X}^{-1}(\lambda \oplus \gamma)$. On account of the adjoint-equivariance (7.4), the level manifold $J^{-1}(\lambda \oplus \gamma)$ admits the action of the isotropy subgroup

$$
G_{\lambda \oplus \gamma}=\left\{(g, h) \in \mathrm{SO}(n) \times \mathrm{SO}(n) \mid \operatorname{Ad}_{(g, h)}(\lambda \oplus \gamma)=\lambda \oplus \gamma\right\}
$$

Further, another isotropy subgroup $G_{X} \cong G_{A} \cong\left(\mathbf{Z}_{2}\right)^{n-1}$ acts on $J^{-1}(\lambda \oplus \gamma)$ as well (see (6.2) for the definition of $G_{A}$ ).

According to the singular value decomposition $X=R A S^{-1}$, we now express the elements of $\left.J^{-1}(\lambda \oplus \gamma)\right|_{T^{*}(\dot{\mathcal{P}})}$ as

$$
\left(R A S^{-1}, R\left(\xi^{\prime} A+Y^{\prime}+A \eta^{\prime \top}\right) S^{-1}\right),
$$

where $Y^{\prime} \in H_{A}$ which is determined by $Y=R Y^{\prime} S^{-1}$, and where $\xi^{\prime}$ and $\eta^{\prime}$ are given in (7.9). Then, under the action of $(g, h) \in G_{\lambda \oplus \gamma}$, the element (7.12) transforms to

$$
\left(g R A(h S)^{-1}, g R\left(\xi^{\prime} A+Y^{\prime}+A \eta^{\prime \top}\right)(h S)^{-1}\right) .
$$

Moreover, the isotropy subgroup $G_{A}$ acts on $\left.J^{-1}(\lambda \oplus \gamma)\right|_{T^{*}(\dot{\mathcal{P}})}$ through $D A D=A$ with $D \in G_{A}$. From these facts, we observe that $\left\{\xi^{\prime} A+Y^{\prime}+A \eta^{\prime \top} \mid Y^{\prime} \in H_{A}\right\} \simeq H_{A} \simeq$ $T_{\pi(A)}(G \backslash \dot{\mathcal{P}}) \simeq T_{\pi(A)}^{*}(G \backslash \dot{\mathcal{P}})$, and that the $G_{\lambda \oplus \gamma} \times\left(\mathbf{Z}_{2}\right)^{n-1}$ action defines an equivalence relation on $\mathrm{SO}(n) \times \mathrm{SO}(n)$ through $(R, S) \sim(g R D, h S D)$. Let $\mathcal{O}_{\lambda \oplus \gamma}$ denote the adjoint orbit through $\lambda \oplus \gamma$ in $\mathfrak{s o}(n) \oplus \mathfrak{s o}(n)$. Then, the factor space of $\mathrm{SO}(n) \times \mathrm{SO}(n)$ by $G_{\lambda \oplus \gamma} \times\left(\mathbf{Z}_{2}\right)^{n-1}$ is expressed as $\mathcal{O}_{\lambda \oplus \gamma} /\left(\mathbf{Z}_{2}\right)^{n-1}$. Hence, the factor space of $\left.J^{-1}(\lambda \oplus \gamma)\right|_{T^{*}(\dot{\mathcal{P}})}$ by $G_{\lambda \oplus \gamma} \times\left(\mathbf{Z}_{2}\right)^{n-1}$ is put in the form

$$
\left.\left(G_{\lambda \oplus \gamma} \times\left(\mathbf{Z}_{2}\right)^{n-1}\right) \backslash J^{-1}(\lambda \oplus \gamma)\right|_{T^{*}(\dot{\mathcal{P}})} \simeq T^{*}(G \backslash \dot{\mathcal{P}}) \times_{G \backslash \dot{\mathcal{P}}}\left(\widetilde{\mathcal{O}}_{\lambda \oplus \gamma} /\left(\mathbf{Z}_{2}\right)^{n-1}\right)
$$

where $\widetilde{\mathcal{O}}_{\lambda \oplus \gamma} /\left(\mathbf{Z}_{2}\right)^{n-1}$ denotes a fiber bundle over $G \backslash \dot{\mathcal{P}}$ whose fiber is $\mathcal{O}_{\lambda \oplus \gamma} /\left(\mathbf{Z}_{2}\right)^{n-1}$.

The $T^{*}(G \backslash \dot{\mathcal{P}}) \times_{G \backslash \dot{\mathcal{P}}}\left(\widetilde{\mathcal{O}}_{\lambda \oplus \gamma} /\left(\mathbf{Z}_{2}\right)^{n-1}\right)$ should be a symplectic leaf of $G \backslash T^{*}(\dot{\mathcal{P}})$ from the orbit reduction theorem [10]. Though the orbit reduction theorem and the cotangent bundle reduction theorem are proved on the assumption that the configuration space $\mathcal{P}^{\prime}$ be a principal fiber bundle with structure group $G^{\prime}$ acting freely on it, those theorems will hold true on the assumption that $G^{\prime}$ has the same orbit type on $\mathcal{P}^{\prime}$ and that the isotropy subgroup is a discrete finite group. This is because $G^{\prime} \backslash \mathcal{P}^{\prime}$ is a manifold, and 
because differential calculus for symplectic forms in those theorems will not be affected under the relaxed assumption stated above.

In what follows, we work with the reduced system. Let $P=\xi X+Y+X \eta^{\top}$ with $X \in \dot{\mathcal{P}}$. Then, like (7.5), one has

$$
\left(\begin{array}{c}
\xi \\
\eta
\end{array}\right)=\left(\begin{array}{cc}
\mathcal{A}_{X}^{L} & -2 \mathrm{Ad}_{X} \\
-2 \operatorname{Ad}_{X}^{\top} & \mathcal{A}_{X}^{R}
\end{array}\right)^{-1}\left(\begin{array}{c}
L \\
\Gamma
\end{array}\right) .
$$

With this in mind, we calculate the canonical one-form $\theta$,

$$
\begin{aligned}
\theta & =\left\langle\xi X+Y+X \eta^{\top}, d X\right\rangle \\
& =\langle Y, d X\rangle+\left\langle\left(\begin{array}{c}
\xi \\
\eta
\end{array}\right),\left(\begin{array}{c}
d X X^{\top}-X d X^{\top} \\
d X^{\top} X-X^{\top} d X
\end{array}\right)\right\rangle_{\mathfrak{s o}(n) \oplus \mathfrak{s o}(n)} \\
& =\langle Y, d X\rangle+\left\langle\left(\begin{array}{cc}
\mathcal{A}_{X}^{L} & -2 \mathrm{Ad}_{X} \\
-2 \mathrm{Ad}_{X}^{\top} & \mathcal{A}_{X}^{R}
\end{array}\right)^{-1}\left(\begin{array}{c}
L \\
\Gamma
\end{array}\right),\left(\begin{array}{c}
d X X^{\top}-X d X^{\top} \\
d X^{\top} X-X^{\top} d X
\end{array}\right)\right\rangle_{\mathfrak{s o}(n) \oplus \mathfrak{s o}(n)} \\
& =\langle Y, d X\rangle+\left\langle L \oplus \Gamma, \omega_{X}^{B}\right\rangle_{\mathfrak{s o}(n) \oplus \mathfrak{s o}(n)},
\end{aligned}
$$

where we have used the fact that $\mathcal{B}_{X}^{-1}$ is a symmetric operator, and the definition of $\omega^{B}$. Let $\iota_{\lambda \oplus \gamma}:\left.J^{-1}(\lambda \oplus \gamma)\right|_{T^{*}(\dot{\mathcal{P}})} \rightarrow T^{*}(\dot{\mathcal{P}})$ be the inclusion map. Then, we obtain, from $(7.16)$,

$$
\iota_{\lambda \oplus \gamma}^{*} \theta=\langle Y, d X\rangle+\left\langle\lambda \oplus \gamma, \omega_{X}^{B}\right\rangle_{\mathfrak{s o}(n) \oplus \mathfrak{s o}(n)} .
$$

In a similar manner, the kinetic energy is brought into the form

$$
\begin{aligned}
\mathcal{K} & =\frac{1}{2}\left\langle\xi X+Y+X \eta^{\top}, \xi X+Y+X \eta^{\top}\right\rangle \\
& =\frac{1}{2}\langle Y, Y\rangle+\frac{1}{2}\left\langle\left(\begin{array}{c}
\xi \\
\eta
\end{array}\right),\left(\begin{array}{cc}
\mathcal{A}_{X}^{L} & -2 \operatorname{Ad}_{X} \\
-2 \operatorname{Ad}_{X}^{\top} & \mathcal{A}_{X}^{R}
\end{array}\right)\left(\begin{array}{c}
\xi \\
\eta
\end{array}\right)\right\rangle_{\mathfrak{s o}(n) \oplus \mathfrak{s o}(n)} \\
& =\frac{1}{2}\langle Y, Y\rangle+\frac{1}{2}\left\langle\left(\begin{array}{cc}
\mathcal{A}_{X}^{L} & -2 \operatorname{Ad}_{X} \\
-2 \operatorname{Ad}_{X}^{\top} & \mathcal{A}_{X}^{R}
\end{array}\right)^{-1}\left(\begin{array}{c}
L \\
\Gamma
\end{array}\right),\left(\begin{array}{c}
L \\
\Gamma
\end{array}\right)\right\rangle_{\mathfrak{s o}(n) \oplus \mathfrak{s o}(n)}
\end{aligned}
$$

It then follows that

$$
\iota_{\lambda \oplus \gamma}^{*} \mathcal{H}=\frac{1}{2}\langle Y, Y\rangle+\frac{1}{2}\left\langle\mathcal{B}_{X}^{-1}(\lambda \oplus \gamma), \lambda \oplus \gamma\right\rangle_{\mathfrak{s o}(n) \oplus \mathfrak{s o}(n)}+\mathcal{U} .
$$

Theorem 7.1. Let $\left(T^{*}(\mathcal{P}), d \theta, \mathcal{H}\right)$ be the Hamiltonian system for a pseudo-rigid body, where $\theta$ and $\mathcal{H}$ are of the same form as given in (3.21) and (3.22), respectively, and 
where $\mathcal{U}$ is assumed to be invariant under the $\mathrm{SO}(n) \times \mathrm{SO}(n)$ action. The system admits $\mathrm{SO}(n) \times \mathrm{SO}(n)$ symmetry and have the associated moment map $J=L \oplus \Gamma$, where $L$ and $\Gamma$ are the angular momentum and the circulation, respectively, and given in (7.3). For $\lambda \oplus \gamma \in \mathfrak{s o}(n) \oplus \mathfrak{s o}(n) \backslash\{0 \oplus 0\}$, the reduced phase space $\left(G_{\lambda \oplus \gamma} \times(\mathbf{Z})^{n-1}\right) \backslash J^{-1}(\lambda \oplus$ $\gamma)\left.\right|_{T^{*}(\dot{\mathcal{P}})}$ is expressed as in $(7.14)$, and the reduced symplectic form $\omega_{\lambda \oplus \gamma}$ and the reduced Hamiltonian $\mathcal{H}_{\lambda \oplus \gamma}$ are determined through $\iota_{\lambda \oplus \gamma}^{*} d \theta=\pi_{\lambda \oplus \gamma}^{*} \omega_{\lambda \oplus \gamma}$ and $\iota_{\lambda \oplus \gamma}^{*} \mathcal{H}=\pi_{\lambda \oplus \gamma}^{*} \mathcal{H}$, where $\iota_{\lambda \oplus \gamma}^{*} \theta$ and $\iota_{\lambda \oplus \gamma}^{*} \mathcal{H}$ are given in (7.17) and (7.19), respectively, and where $\pi_{\lambda \oplus \gamma}$ is the projection of $\left.J^{-1}(\lambda \oplus \gamma)\right|_{T^{*}(\dot{\mathcal{P}})}$ onto the reduced phase space.

\subsection{The local expression of the reduced system}

We wish to describe the reduced system in terms of local coordinates. We start by expressing the canonical one-form $\theta$ in terms of local coordinates. Equation (7.16) along with $X=R A S^{-1}$ is rewritten as

$$
\theta=\left\langle p_{A}, d A\right\rangle+\left\langle\left(\begin{array}{c}
p_{\Omega} \\
p_{\Lambda}
\end{array}\right),\left(\begin{array}{c}
R^{-1} d R \\
S^{-1} d S
\end{array}\right)\right\rangle_{\mathfrak{s o}(n) \oplus \mathfrak{s o}(n)},
$$

where (6.26) and (6.29) have been used, and where

$$
p_{A}=R^{-1} Y S \text {. }
$$

We here note that if $A \in \dot{\mathcal{P}}$, we may put $R^{-1} Y S=\dot{A}=\operatorname{diag}\left(\dot{a}_{1}, \cdots, \dot{a}_{n}\right) \in H_{A}$ on account of (6.7), so that $p_{A}=\dot{A}=\partial \mathcal{K} / \partial \dot{A}$, where $\mathcal{K}$ is given in (6.28). The exterior derivative of $\theta$ is then shown to be expressed as

$$
\begin{aligned}
d \theta= & \left\langle d p_{A} \wedge d A\right\rangle+\left\langle\left(\begin{array}{c}
d p_{\Omega} \\
d p_{\Lambda}
\end{array}\right) \wedge\left(\begin{array}{c}
R^{-1} d R \\
S^{-1} d S
\end{array}\right)\right\rangle_{\mathfrak{s o}(n) \oplus \mathfrak{s o}(n)} \\
& -\left\langle\left(\begin{array}{c}
p_{\Omega} \\
p_{\Lambda}
\end{array}\right),\left(\begin{array}{c}
R^{-1} d R \wedge R^{-1} d R \\
S^{-1} d S \wedge S^{-1} d S
\end{array}\right)\right\rangle_{\mathfrak{s o}(n) \oplus \mathfrak{s o}(n)}
\end{aligned}
$$

where $\langle\cdot \wedge \cdot\rangle$ denotes an inner product combined with the wedge product; $\langle a \wedge b\rangle=$ $\sum a_{i j} \wedge b_{i j}$ with $a=\left(a_{i j}\right), b=\left(b_{i j}\right)$.

For a generic Hamiltonian $\mathcal{H}$, the Hamiltonian vector field $X_{\mathcal{H}}$ is determined through $\iota\left(X_{\mathcal{H}}\right) d \theta=-d \mathcal{H}$, where $\iota$ denote the interior product. The Hamilton equations of motion determined through $X_{\mathcal{H}}$ then turn out to be the same as (4.17). For a pseudo-rigid body, the Hamiltonian is given by (6.30). 
We proceed to the reduced Hamilton equations for the pseudo-rigid body. If restricted on $T^{*}(G \backslash \dot{\mathcal{P}}) \times_{G \backslash \dot{\mathcal{P}}}\left(\widetilde{\mathcal{O}}_{\lambda \oplus \gamma} /\left(\mathbf{Z}_{2}\right)^{n-1}\right), p_{\Omega}$ and $p_{\Lambda}$ are reduced, from (6.29), to

$$
\left(p_{\Omega} \oplus p_{\Lambda}\right)^{\mathrm{red}}=\operatorname{Ad}_{(R, S)}^{-1}(\lambda \oplus \gamma)
$$

which means that if restricted, $p_{\Omega} \oplus p_{\Lambda}$ is sitting on the (co)adjoint orbit of $\operatorname{SO}(n) \times$ $\mathrm{SO}(n)$ through $\lambda \oplus \gamma \in \mathfrak{s o}(n) \oplus \mathfrak{s o}(n)$. From (6.30) and (7.23), the reduced Hamiltonian is expressed as

$$
\mathcal{H}_{\lambda \oplus \gamma}=\frac{1}{2}\left\langle p_{A}, p_{A}\right\rangle+\frac{1}{2}\left\langle\operatorname{Ad}_{(R, S)}^{-1}(\lambda \oplus \gamma), \mathcal{B}_{A}^{-1}\left(\operatorname{Ad}_{(R, S)}^{-1}(\lambda \oplus \gamma)\right)\right\rangle_{\mathfrak{s o}(n) \oplus \mathfrak{s o}(n)}+\mathcal{U}(A)
$$

Among the Hamilton equations of motion (4.18), the second and the last of them are put together to take the form

$$
\frac{d}{d t}\left(\begin{array}{c}
p_{\Omega} \\
p_{\Lambda}
\end{array}\right)=\left[\left(\begin{array}{c}
p_{\Omega} \\
p_{\Lambda}
\end{array}\right),\left(\begin{array}{cc}
\mathcal{A}_{A}^{L} & -2 \mathrm{Ad}_{A} \\
-2 \mathrm{Ad}_{A} & \mathcal{A}_{A}^{R}
\end{array}\right)^{-1}\left(\begin{array}{c}
p_{\Omega} \\
p_{\Lambda}
\end{array}\right)\right],
$$

where (6.29) has been used and where the commutator on $\mathfrak{s o}(n) \oplus \mathfrak{s o}(n)$ is defined as

$$
\left[\left(\begin{array}{c}
\xi^{\prime} \\
\eta^{\prime}
\end{array}\right),\left(\begin{array}{c}
\xi \\
\eta
\end{array}\right)\right]=\left(\begin{array}{l}
{\left[\xi^{\prime}, \xi\right]} \\
{\left[\eta^{\prime}, \eta\right]}
\end{array}\right)
$$

If $p_{\Omega} \oplus p_{\Lambda}$ is replaced by $\left(p_{\Omega} \oplus p_{\Lambda}\right)^{\text {red }}$ together with (7.23), Eq. (7.25) is viewed as the reduced equation on the orbit bundle $\widetilde{\mathcal{O}}_{\lambda \oplus \gamma} /\left(\mathbf{Z}_{2}\right)^{n-1}$,

$$
\frac{d}{d t} \operatorname{Ad}_{(R, S)}^{-1}(\lambda \oplus \gamma)=\left[\operatorname{Ad}_{(R, S)}^{-1}(\lambda \oplus \gamma), \mathcal{B}_{A}^{-1}\left(\operatorname{Ad}_{(R, S)}^{-1}(\lambda \oplus \gamma)\right)\right]
$$

The remaining Hamilton equations reduce to

$$
\frac{d A}{d t}=p_{A}, \quad \frac{d}{d t} p_{A}=-\frac{\partial \mathcal{H}_{\lambda \oplus \gamma}}{\partial A} .
$$

Equations (7.27) and (7.28) form the reduced Hamilton equations on the reduced phase space. See [22] for the case of $n=3$.

\section{Relative equilibria}

We now consider critical points of the reduced Hamilton equations (7.27) and (7.28). Let $\left(A, p_{A}, \operatorname{Ad}_{(R, S)}^{-1}(\lambda \oplus \gamma)\right)=\left(A_{0}, 0, \kappa \oplus \mu\right)$ be a critical point of them in the reduced 
phase space. In other words, $\partial \mathcal{H}_{\lambda \oplus \gamma} / \partial A$ vanishes at $\left(A_{0}, 0, \kappa \oplus \mu\right)$ and $\kappa \oplus \mu$ satisfies the condition $\left[\kappa \oplus \mu, \mathcal{B}_{A_{0}}^{-1}(\kappa \oplus \mu)\right]=0$. A sufficient condition for the latter is that $\kappa \oplus \mu$ is an eigenvector of the symmetric operator $\mathcal{B}_{A_{0}}^{-1}$. Equation (6.27) with $\Omega=R^{-1} \dot{R}, \Lambda=$ $S^{-1} \dot{S}, L=\lambda$, and $\Gamma=\gamma$ gives rise to the equations for $R$ and $S$,

$$
\left(\begin{array}{c}
R^{-1} \dot{R} \\
S^{-1} \dot{S}
\end{array}\right)=\left(\begin{array}{cc}
\mathcal{A}_{A_{0}}^{L} & -2 \mathrm{Ad}_{A_{0}} \\
-2 \mathrm{Ad}_{A_{0}} & \mathcal{A}_{A_{0}}^{R}
\end{array}\right)^{-1}\left(\begin{array}{c}
\kappa \\
\mu
\end{array}\right) .
$$

The right-hand side of the above equation is expressed as $\mathcal{B}_{A_{0}}^{-1}(\kappa \oplus \mu)$. On account of (7.9), there exist $\kappa^{\prime}$ and $\mu^{\prime}$ such that $\kappa^{\prime} \oplus \mu^{\prime}:=\mathcal{B}_{A_{0}}^{-1}(\kappa \oplus \mu)$. Then, Eq. (8.1) are easily integrated to give a solution of the form

$$
R(t)=R_{0} \exp \left(t \kappa^{\prime}\right), \quad S(t)=S_{0} \exp \left(t \mu^{\prime}\right)
$$

Hence, the pseudo-rigid body (or a Riemann ellipsoid) is in relative equilibrium,

$$
X(t)=\exp \left(t \operatorname{Ad}_{R_{0}} \kappa^{\prime}\right) X_{0} \exp \left(-t \operatorname{Ad}_{S_{0}} \mu^{\prime}\right),
$$

where $X_{0}=R_{0} A_{0} S_{0}^{-1}$. We here note that the condition $\left[\kappa \oplus \mu, \mathcal{B}_{A_{0}}^{-1}(\kappa \oplus \mu)\right]=0$ is now rewritten as $\left[\mathcal{B}_{A_{0}}\left(\kappa^{\prime} \oplus \mu^{\prime}\right), \kappa^{\prime} \oplus \mu^{\prime}\right]=0$.

Conversely, we assume that $(X(t), P(t))$ with $X(t)$ given above and $P(t)=\dot{X}(t)$ is a solution to the Hamilton equations (4.18), where $R(t)=R_{0} \exp \left(t \kappa^{\prime}\right), S(t)=$ $S_{0} \exp \left(t \mu^{\prime}\right)$, and $A(t)=A_{0}$. Then, from (2.32) and (6.9) along with $\Omega=\kappa^{\prime}$ and $\Lambda=\mu^{\prime}$, the conserved momentum $J=L \oplus \Gamma$ proves to take the value

$$
\lambda \oplus \gamma:=\operatorname{Ad}_{\left(R_{0}, S_{0}\right)} \mathcal{B}_{A_{0}}\left(\kappa^{\prime} \oplus \mu^{\prime}\right)
$$

On the other hand, from (8.3) and $P(t)=\exp \left(t \operatorname{Ad}_{R_{0}} \kappa^{\prime}\right) P_{0} \exp \left(-t \operatorname{Ad}_{S_{0}} \mu^{\prime}\right)$ with $P_{0}=$ $\operatorname{Ad}_{R_{0}}\left(\kappa^{\prime}\right) X_{0}-X_{0} \operatorname{Ad}_{S_{0}}\left(\mu^{\prime}\right)$, the adjoint-equivariance (7.4) of $J$ implies that $\operatorname{Ad}_{\exp \left(\operatorname{tad}_{R_{0}} \kappa^{\prime}\right)} \oplus$ $\operatorname{Ad}_{\exp \left(t \operatorname{Ad}_{S_{0}} \mu^{\prime}\right)}$ leaves $\lambda \oplus \gamma$ invariant, so that $\left.\operatorname{Ad}_{\left(\exp \left(t \operatorname{Ad}_{R_{0}} \kappa^{\prime}\right), \exp \left(t \operatorname{Ad}_{S_{0}} \mu^{\prime}\right)\right.}\right) \in G_{\lambda \oplus \gamma}$. It then follows that $\operatorname{Ad}_{R_{0}} \kappa^{\prime} \oplus \operatorname{Ad}_{S_{0}} \mu^{\prime} \in \mathcal{G}_{\lambda \oplus \gamma} ;\left[\operatorname{Ad}_{\left(R_{0}, S_{0}\right)}\left(\kappa^{\prime} \oplus \mu^{\prime}\right), \lambda \oplus \gamma\right]=0$. From this fact along with (8.4), one obtains

$$
\begin{aligned}
& \operatorname{Ad}_{\left(R_{0}, S_{0}\right)}\left[\kappa^{\prime} \oplus \mu^{\prime}, \mathcal{B}_{A_{0}}\left(\kappa^{\prime} \oplus \mu^{\prime}\right)\right] \\
= & {\left[\operatorname{Ad}_{\left(R_{0}, S_{0}\right)}\left(\kappa^{\prime} \oplus \mu^{\prime}\right), \operatorname{Ad}_{\left(R_{0}, S_{0}\right)} \mathcal{B}_{A_{0}}\left(\kappa^{\prime} \oplus \mu^{\prime}\right)\right]=0, }
\end{aligned}
$$


and hence

$$
\left[\kappa^{\prime} \oplus \mu^{\prime}, \mathcal{B}_{A_{0}}\left(\kappa^{\prime} \oplus \mu^{\prime}\right)\right]=0 .
$$

From (8.4), Eq. (8.6) is brought into

$$
\left[\operatorname{Ad}_{\left(R_{0}, S_{0}\right)}^{-1}(\lambda \oplus \gamma), \mathcal{B}_{A_{0}}^{-1} \operatorname{Ad}_{\left(R_{0}, S_{0}\right)}^{-1}(\lambda \oplus \gamma)\right]=0
$$

We now turn to the reduced Hamilton equations. The above equation implies that the right-hand side of (7.27) vanishes at $\operatorname{Ad}_{\left(R_{0}, S_{0}\right)}^{-1}(\lambda \oplus \gamma)$. Further, from $A(t)=A_{0}$, one has $p_{A}=0$. Since the solution of the Hamilton equations projects to that of the reduced Hamilton equations, $\partial \mathcal{H}_{\lambda \oplus \gamma} / \partial A$, the right-hand side of (7.28), must vanish at $\left(A_{0}, 0, \operatorname{Ad}_{\left(R_{0}, S_{0}\right)}^{-1}(\lambda \oplus \gamma)\right)$. Thus, $\left(A_{0}, 0, \operatorname{Ad}_{\left(R_{0}, S_{0}\right)}^{-1}(\lambda \oplus \gamma)\right)$ gives a critical point of the reduced Hamilton equations. Thus we have shown the following.

Proposition 8.1. The curve $X(t)$ given in (8.3) with $A(t)=A_{0}$ is in relative equilibrium, if and only if Eq. (8.6) holds and $\partial \mathcal{H}_{\lambda \oplus \gamma} / \partial A$ vanishes at $\left(A_{0}, 0, \operatorname{Ad}_{\left(R_{0}, S_{0}\right)}^{-1}(\lambda \oplus \gamma)\right)$ in the reduced phase space. The condition (8.6) is also equivalent to $\operatorname{Ad}_{R_{0}} \kappa^{\prime} \oplus \operatorname{Ad}_{S_{0}} \mu^{\prime} \in$ $\mathcal{G}_{\lambda \oplus \gamma}$, where $\mathcal{G}_{\lambda \oplus \gamma}$ denotes the Lie algebra of the isotropy subgroup $G_{\lambda \oplus \mu}$ given in (7.11). Relative equilibrium solutions $(X(t), P(t))$ to the Hamilton equations and critical points of the reduced Hamilton equations are in one-to-one correspondence.

We now show that the point $\left(A_{0}, \operatorname{Ad}_{\left(R_{0}, S_{0}\right)}^{-1}(\lambda \oplus \gamma)\right)$ coming from the critical point mentioned in Prop. 8.1 is characterized as a critical point of the amended potential defined on the orbit bundle $\widetilde{\mathcal{O}}_{\lambda \oplus \gamma} /\left(\mathbf{Z}_{2}\right)^{n-1}$ to be

$$
\mathcal{V}_{\lambda \oplus \gamma}\left(A, \operatorname{Ad}_{(R, S)}^{-1}(\lambda \oplus \gamma)\right):=\mathcal{U}(A)+\frac{1}{2}\left\langle\operatorname{Ad}_{(R, S)}^{-1}(\lambda \oplus \gamma), \mathcal{B}_{A}^{-1}\left(\operatorname{Ad}_{(R, S)}^{-1}(\lambda \oplus \gamma)\right)\right\rangle_{\mathfrak{s o}(n) \oplus \mathfrak{s o}(n)}
$$

From Prop. 8.1, we see that $\partial \mathcal{V}_{\lambda \oplus \gamma} / \partial A=0$ at $\left(A_{0}, \operatorname{Ad}_{\left(R_{0}, S_{0}\right)}^{-1}(\lambda \oplus \gamma)\right)$, since $\mathcal{V}_{\lambda \oplus \gamma}=$ $\mathcal{H}_{\lambda \oplus \gamma}-\frac{1}{2}\left\langle p_{A}, p_{A}\right\rangle$ and $p_{A}=0$. We consider the derivative of $\mathcal{V}_{\lambda \oplus \gamma}$ with respect to the variable $\operatorname{Ad}_{(R, S)}^{-1}(\lambda \oplus \gamma)$ at $(R, S)=\left(R_{0}, S_{0}\right)$. Let $\zeta_{1} \oplus \zeta_{2}$ be an arbitrary element of $\mathfrak{s o}(n) \oplus \mathfrak{s o}(n)$. Then, any tangent vector at $\operatorname{Ad}_{\left(R_{0}, S_{0}\right)}^{-1}(\lambda \oplus \gamma)$ takes the form

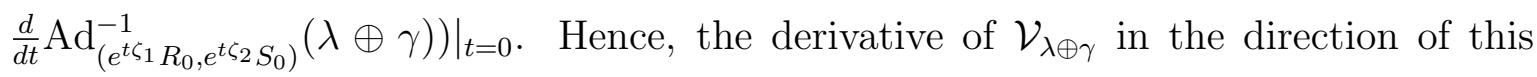


tangent vector is expressed and calculated as

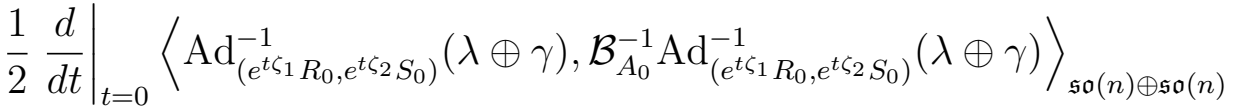

$$
\begin{aligned}
& =\left.\frac{1}{2} \frac{d}{d t}\right|_{t=0}\left\langle\operatorname{Ad}_{\left(R_{0}, S_{0}\right)}^{-1} \operatorname{Ad}_{\left(e^{\left.t \zeta_{1}, e^{t \zeta_{2}}\right)}\right.}^{-1}(\lambda \oplus \gamma), \mathcal{B}_{A_{0}}^{-1} \operatorname{Ad}_{\left(R_{0}, S_{0}\right)}^{-1} \operatorname{Ad}_{\left(e^{\left.t \zeta_{1}, e^{t \zeta_{2}}\right)}\right.}^{-1}(\lambda \oplus \gamma)\right\rangle_{\mathfrak{s o}(n) \oplus \mathfrak{s o}(n)} \\
& =-\left\langle\operatorname{Ad}_{\left(R_{0}, S_{0}\right)}^{-1}\left[\zeta_{1} \oplus \zeta_{2}, \lambda \oplus \gamma\right], \mathcal{B}_{A_{0}}^{-1} \operatorname{Ad}_{\left(R_{0}, S_{0}\right)}^{-1}(\lambda \oplus \gamma)\right\rangle_{\mathfrak{s o}(n) \oplus \mathfrak{s o}(n)} \\
& =-\left\langle\left[\operatorname{Ad}_{\left(R_{0}, S_{0}\right)}^{-1}\left(\zeta_{1} \oplus \zeta_{2}\right), \operatorname{Ad}_{\left(R_{0}, S_{0}\right)}^{-1}(\lambda \oplus \gamma)\right], \mathcal{B}_{A_{0}}^{-1} \operatorname{Ad}_{\left(R_{0}, S_{0}\right)}^{-1}(\lambda \oplus \gamma)\right\rangle_{\mathfrak{s o}(n) \oplus \mathfrak{s o}(n)} \\
& =-\left\langle\operatorname{Ad}_{\left(R_{0}, S_{0}\right)}^{-1}\left(\zeta_{1} \oplus \zeta_{2}\right),\left[\operatorname{Ad}_{\left(R_{0}, S_{0}\right)}^{-1}(\lambda \oplus \gamma), \mathcal{B}_{A_{0}}^{-1} \operatorname{Ad}_{\left(R_{0}, S_{0}\right)}^{-1}(\lambda \oplus \gamma)\right]\right\rangle_{\mathfrak{s o}(n) \oplus \mathfrak{s o}(n)} \\
& =0 \text {, }
\end{aligned}
$$

where we have used (8.7) and the formula

$$
\left\langle\left[\xi_{1} \oplus \xi_{2}, \eta_{1} \oplus \eta_{2}\right], \zeta_{1} \oplus \zeta_{2}\right\rangle_{\mathfrak{s o}(n) \oplus \mathfrak{s o}(n)}=\left\langle\xi_{1} \oplus \xi_{2},\left[\eta_{1} \oplus \eta_{2}, \zeta_{1} \oplus \zeta_{2}\right]\right\rangle_{\mathfrak{s o}(n) \oplus \mathfrak{s o}(n)}
$$

Since $\zeta_{1} \oplus \zeta_{2}$ is arbitrary, the above equation implies that the derivative of $\mathcal{V}_{\lambda \oplus \gamma}$ with respect to the orbit bundle variables $\operatorname{Ad}_{(R, S)}^{-1}(\lambda \oplus \gamma)$ vanishes at $\left(R_{0}, S_{0}\right)$.

Proposition 8.2. Critical points of the reduced Hamilton equations are determined by critical points of the amended potential (8.8) defined on the orbit bundle $\widetilde{\mathcal{O}}_{\lambda \oplus \gamma} /\left(\mathbf{Z}_{2}\right)^{n-1}$.

We notice in addition that the amended potential on $\dot{\mathcal{P}}$ is defined to be

$$
\widetilde{\mathcal{V}}_{\lambda \oplus \gamma}(X):=\mathcal{U}(X)+\frac{1}{2}\left\langle\lambda \oplus \gamma, \mathcal{B}_{X}^{-1}(\lambda \oplus \gamma)\right\rangle_{\mathfrak{s o}(n) \oplus \mathfrak{s o}(n)},
$$

which is an extension of (8.8). From the assumption that $\mathcal{U}(X)$ is $G$-invariant and from the transformation property of $\mathcal{B}_{X}$, we see that $\widetilde{\mathcal{V}}_{\lambda \oplus \gamma}(X)$ takes the same values as those $\mathcal{V}_{\lambda \oplus \gamma}\left(A, \operatorname{Ad}_{(R, S)}^{-1}(\lambda \oplus \gamma)\right)$ does. Hence, the relative equilibrium is also characterized by critical points of the amended potential (8.11).

For comparison's sake, we mention relative equilibria in the Lagrangian formalism. According to the relative equilibrium theory [21], $\left(X_{0}, \dot{X}_{0}\right)$ with $\dot{X}_{0}=\xi X_{0}+X_{0} \eta^{\top}$ is a relative equilibrium or the curve $X(t)=e^{t \xi} X_{0} e^{-t \eta}$ is a solution to the Euler-Lagrange equation, if and only if $X_{0}$ is a critical point of the augmented potential. In the present case, the Euler-Lagrange equations are given in Prop. 4.2, and the augmented potential takes the form

$$
\mathcal{U}_{\xi \oplus \eta}(X):=\mathcal{U}(X)-\frac{1}{2}\left\langle\xi \oplus \eta, \mathcal{B}_{X}(\xi \oplus \eta)\right\rangle_{\mathfrak{s o}(n) \oplus \mathfrak{s o}(n)},
$$


where the second term of the right-hand side of the above comes from the second term of the right-hand side of (7.18). In fact, we can verify this fact by using Prop. 4.2. We note here that the Euler-Lagrange equations in Prop. 4.2 are now rewritten as

$$
\begin{aligned}
& \frac{d}{d t} \dot{A}=\frac{\partial}{\partial A}(\mathcal{K}-\mathcal{U}), \\
& \frac{d}{d t} \mathcal{B}_{A}(\Omega \oplus \Lambda)=\left[\mathcal{B}_{A}(\Omega \oplus \Lambda), \Omega \oplus \Lambda\right] .
\end{aligned}
$$

In the rest of this section, we look into Eq. (8.6). As is easily seen, this equation is satisfied by eigenvectors of the symmetric operator $\mathcal{B}_{A_{0}}$, which are easily found by calculation. Let $\xi=\left(\xi_{i j}\right)$. Then, $\mathcal{A}_{A_{0}}^{L}(\xi)=\mathcal{A}_{A_{0}}^{R}(\xi)=\left(\left(a_{i}^{2}+a_{j}^{2}\right) \xi_{i j}\right)$ and $\operatorname{Ad}_{A_{0}}(\xi)=$ $\operatorname{Ad}_{A_{0}}^{\top}(\xi)=\left(a_{i} a_{j} \xi_{i j}\right)$. Hence, we have

$$
\mathcal{B}_{A_{0}}\left(\begin{array}{c}
\xi \\
\eta
\end{array}\right)=\left(\begin{array}{c}
\left(a_{i}^{2}+a_{j}^{2}\right) \xi_{i j}-2 a_{i} a_{j} \eta_{i j} \\
-2 a_{i} a_{j} \xi_{i j}+\left(a_{i}^{2}+a_{j}^{2}\right) \eta_{i j}
\end{array}\right)
$$

Let $e_{i j}$ with $i<j$ be the standard basis of $\mathfrak{s o}(n)$, i.e., the $(i, j)$ and $(j, i)$ components of $e_{i j}$ are 1 and -1 , respectively, and the others vanish. Then, the above equation provides

$$
\mathcal{B}_{A_{0}}\left(\begin{array}{c}
e_{i j} \\
e_{i j}
\end{array}\right)=\left(a_{i}-a_{j}\right)^{2}\left(\begin{array}{c}
e_{i j} \\
e_{i j}
\end{array}\right), \quad \mathcal{B}_{A_{0}}\left(\begin{array}{c}
e_{i j} \\
-e_{i j}
\end{array}\right)=\left(a_{i}+a_{j}\right)^{2}\left(\begin{array}{c}
e_{i j} \\
-e_{i j}
\end{array}\right)
$$

which shows that $e_{i j} \oplus e_{i j}$ and $e_{i j} \oplus\left(-e_{i j}\right)$ are eigenvectors of $\mathcal{B}_{A_{0}}$, where $i<j$. Since $\operatorname{dim}(\mathfrak{s o}(n) \oplus \mathfrak{s o}(n))=n(n-1)$, and since these vectors are linearly independent, we have found all the eigenvectors for $\mathcal{B}_{A_{0}}$ with $A_{0} \in \dot{\mathcal{P}}$.

The condition (8.6) may be satisfied by vectors other than the eigenvectors of $\mathcal{B}_{A_{0}}$, as is shown below: Let $\lambda$ and $\lambda^{\prime}$ be distinct eigenvalues of $\mathcal{B}_{A_{0}}$ and $\xi \oplus \eta$ and $\xi^{\prime} \oplus \eta^{\prime}$ respective associated eigenvectors; $\mathcal{B}_{A_{0}}(\xi \oplus \eta)=\lambda(\xi \oplus \eta)$ and $\mathcal{B}_{A_{0}}\left(\xi^{\prime} \oplus \eta^{\prime}\right)=\lambda^{\prime}\left(\xi^{\prime} \oplus \eta^{\prime}\right)$ with $\lambda \neq \lambda^{\prime}$. Then, a straightforward calculation provides

$$
\left[\xi \oplus \eta+\xi^{\prime} \oplus \eta^{\prime}, \mathcal{B}_{A_{0}}\left(\xi \oplus \eta+\xi^{\prime} \oplus \eta^{\prime}\right)\right]=\left(\lambda^{\prime}-\lambda\right)\left(\left[\xi, \xi^{\prime}\right] \oplus\left[\eta, \eta^{\prime}\right]\right)
$$

If $\left[\xi, \xi^{\prime}\right]=\left[\eta, \eta^{\prime}\right]=0$, the right-hand side of the above equation vanishes, so that (8.6) is satisfied by $\left(\xi+\xi^{\prime}\right) \oplus\left(\eta+\eta^{\prime}\right)$. Incidentally, for the standard basis $e_{i j}$ of $\mathfrak{s o}(n)$, the commutation relations are given by

$$
\left[e_{i j}, e_{k \ell}\right]=e_{i \ell} \delta_{j k}+e_{j k} \delta_{i \ell}-e_{i k} \delta_{j \ell}-e_{j \ell} \delta_{i k}
$$


Hence, one has $\left[e_{i j}, e_{k \ell}\right]=0$ if $i, j, k, \ell$ are distinct to one another. This occurs if $n \geq 4$. Thus, for a linear combination of the eigenvectors $e_{i j} \oplus e_{i j}$ and $e_{k \ell} \oplus e_{k \ell}$ with $i, j, k, \ell$ distinct to one another, we have, for example,

$$
\left[\left(\begin{array}{c}
e_{i j} \\
e_{i j}
\end{array}\right)+\left(\begin{array}{c}
e_{k \ell} \\
e_{k \ell}
\end{array}\right), \mathcal{B}_{A_{0}}\left(\left(\begin{array}{c}
e_{i j} \\
e_{i j}
\end{array}\right)+\left(\begin{array}{c}
e_{k \ell} \\
e_{k \ell}
\end{array}\right)\right)\right]=\left(\left(a_{k}-a_{\ell}\right)^{2}-\left(a_{i}-a_{j}\right)^{2}\right)\left(\begin{array}{l}
{\left[e_{i j}, e_{k \ell}\right]} \\
{\left[e_{i j}, e_{k \ell}\right]}
\end{array}\right)=0 .
$$

The stability of the Riemann ellipsoid in three dimensions is studied in [22]. As for relative equilibria for the generalized rigid body, see [23], in which the left-invariant Lagrangian system on the tangent bundle of a Lie group is discussed. Proposition 8. 1 is an extension of a proposition [23] on relative equilibrium of the generalized rigid body.

\section{Concluding remarks}

In Sec. 3, we have mentioned that the right $\mathrm{SO}(n)$ action is associated with particle relabeling. In the case of identical $N$ particles in $\mathbf{R}^{n}$, we deals with the set, $\mathbf{R}^{n \times(N-1)}$, of real $n \times(N-1)$ matrices, which is isomorphic with the center-of-mass system. The particle exchange is expressed as the action of the symmetric group $S_{N}$ on $\mathbf{R}^{n \times(N-1)}$ to the right in the form of an irreducible representation of $S_{N}$ in $\mathrm{O}(N-1)$ (see [24] for particle exchange symmetry in quantum mechanics).

In Sec. 3, we have further carried out the reduction procedure for the pseudo-rigid body by the right $\mathrm{SO}(n)$ symmetry in the Lagrangian formalism. However, the Poisson formalism works in the reduction procedure as well. In Ref. [3, 4], the commutation relations (or Poisson structure),

$$
\left\{Q_{i j}, Q_{k \ell}\right\}=0, \quad\left\{Q_{i j}, N_{k \ell}\right\}=\delta_{i \ell} Q_{j k}+\delta_{j \ell} Q_{i k}, \quad\left\{N_{i j}, N_{k \ell}\right\}=\delta_{i \ell} N_{k j}-\delta_{j k} N_{i \ell}
$$

are used to obtain the reduced system on $T(\mathcal{P}) / \mathrm{SO}(n)$. The semi-direct product reduction [25] is performed in three dimensions [4] by using the semi-direct product group $\mathrm{GL}^{+}(3, \mathbf{R}) \ltimes \operatorname{Sym}(3, \mathbf{R})$. Similar model has been studied under the name of collective models [26]. We note that the collective model comes from the idea of collective states for a nucleus model. For example, for the study of nuclear collective states, the $\operatorname{SL}(3, \mathbf{R}) \ltimes \operatorname{Sym}(3, \mathbf{R})$ is treated in $[27,28]$, and the group $\mathrm{O}(3) \times \mathrm{O}(N-1)$ acting on 
$\mathbf{R}^{3 \times(N-1)}$ is studied in [29]. There are a variety of groups associated with collective states, which are out of the scope of the present article.

In Sec. 4, we have used the local coordinates $(R, A, S, \Omega, \dot{A}, \Lambda)$. As is stated in Sec. 6.1, $\dot{A}$ is viewed as the horizontal velocity and $(\Omega, \Lambda)$ as the vertical (angular) velocity with respect to the $\mathrm{SO}(n) \times \mathrm{SO}(n)$ action. Since the bi-connection $\omega^{B}$ is flat, the velocity shift [20] is not needed, and hence the reduced Euler-Lagrange equations look the same as the Hammel equation [20]. In our case, the transformation group is $G=\mathrm{SO}(n) \times \mathrm{SO}(n)$ and the action of $G$ is not free, so that $\dot{\mathcal{P}}$ is not made into a fiber bundle. However, the isotropy subgroup is finite on $\dot{\mathcal{P}}$ and hence the reduction procedure works well with a slight modification.

In Sec. 6, we have treated the isotropy subgroup on the principal stratum $\dot{\mathcal{P}}$. We here write out the isotropy subgroups on the whole $\mathcal{P}$ for $n=3$. Depending on types of singular values, the isotropy subgroups are given by

$$
G_{A} \simeq \begin{cases}\left(\mathbf{Z}_{2}\right)^{2} & \text { for (i) } a_{1}>a_{2}>a_{3}>0, \\ S\left(O(2) \times \mathbf{Z}_{2}\right) & \text { for (ii) } a_{1}=a_{2}>a_{3}, \text { or } a_{1}>a_{2}=a_{3}>0 \\ \mathrm{SO}(3), & \text { for (iii) } a_{1}=a_{2}=a_{3}>0,\end{cases}
$$

where $S\left(O(2) \times \mathbf{Z}_{2}\right)$ denotes the set of matrices of the form $\left(\begin{array}{cc}k & 0 \\ 0 & \varepsilon\end{array}\right)$ with $k \in O(2)$, $\varepsilon= \pm 1$ and $\varepsilon \operatorname{det}(k)=1$. The orbit spaces $G / G_{A}$ are determined accordingly, and the dimensions of the vertical and horizontal subspaces are given, respectively, by

$$
\left(\operatorname{dim} V_{X}, \operatorname{dim} H_{X}\right)= \begin{cases}(6,3) & \text { for (i) } \\ (5,4) & \text { for (ii) } \\ (3,6) & \text { for (iii) }\end{cases}
$$

In general, the group action on the space of rectangular matrices is classified according to types of singular values of matrices. With this idea, the left action of $\mathrm{SO}(3)$ on $\mathbf{R}^{3 \times(N-1)}$ is treated in [30] for the study of multi-particle systems, and the two-sided action of $\mathrm{U}(p) \times \mathrm{U}(q)$ on $\mathbf{C}^{p \times q}$ is studied in relation to multi-qubit entanglement [31].

We have performed the reduction procedure with the right $\mathrm{SO}(n)$ symmetry in Sec. 3. It is needless to say that one can perform the reduction procedure through the left $\mathrm{SO}(n)$ symmetry to obtain a system on $\mathrm{SO}(n) \backslash T(\mathcal{P})$ in the same manner as that for $T(\mathcal{P}) / \mathrm{SO}(n)$. The conservation law associated with the left $\mathrm{SO}(n)$ symmetry 
is the angular momentum. With respect to the left $\mathrm{SO}(n)$ symmetry (with $n=3$ ), the stability of relative equilibria of the pseudo-rigid bodies is extensively studied in [32].

The reduction procedure by the left $\mathrm{SO}(n)$ symmetry has been used in many-body dynamical systems with $n=3$ [33] by using the Guichardet connection, and studied in detail in terms of local coordinates with insight into stratification and boundary behaviors $[34,35]$.

If the reduced system on $T(\mathcal{P}) / \mathrm{SO}(n)$ admits further the left $\mathrm{SO}(n)$ symmetry, we would be able to perform the reduction procedure to obtain a further reduced system on $\mathrm{SO}(n) \backslash T(\mathcal{P}) / \mathrm{SO}(n)$. However, we have difficulty in this stage. Let $Q$ and $N$ be the same as in Sec. 3. They admits the left $\mathrm{SO}(n)$ action, $Q \mapsto g Q g^{-1}, N \mapsto$ $g N g^{-1}$ with $g \in \mathrm{SO}(n)$. However, this action is not free, so that we cannot expect $\mathrm{SO}(n) \backslash T(\mathcal{P}) / \mathrm{SO}(n)$ to be a manifold. However, if we restrict ourselves to $\dot{\mathcal{P}}$, we will be able to obtain a reduced system on $\mathrm{SO}(n) \backslash T(\dot{\mathcal{P}}) / \mathrm{SO}(n)$. The reduced space will be isomorphic with that stated in Th. 7.1. It is a point to make that the action of $\mathrm{SO}(n) \times \mathrm{SO}(n)$ on $\dot{\mathcal{P}}$ and hence on $T(\dot{\mathcal{P}})$ is not free, but the isotropy subgroup is a finite discrete group. See $[11,12]$ for the commuting reduction theorem, which is proved on the assumption that the product Lie group acts freely and properly on the cotangent bundle.

\section{References}

[1] M.E.S. Dias, Acta Appl. Math., 70, 209-230 (2002).

[2] G. Rosensteel and D.J. Rowe, Ann. Phys., 123, 36-60 (1979).

[3] G. Rosensteel, Ann. Phys., 186, 230-291 (1988).

[4] G. Rosensteel, Lett. Math. Phys., 17, 79-86 (1989).

[5] G. Rosensteel, J. Phys. A34, L169-L178 (2001).

[6] S. Chandrasekhar, Ellipsoidal Figures of Equilibrium, Dover, New York, 1987.

[7] J.E. Marsden, Lectures on mechanics, LMS Lecture Note Series, Cambridge University Press, 1992. 
[8] G. Rosensteel, Hamiltonian dynamics of Riemann ellipsoids; in J. Goldstein et al. (eds.), Mathematics applied to science: in memoriam Edward D. Conway, Academic Press, Boston and Tokyo, 1988.

[9] H. Cendra, J.E. Marsden, and T.S. Ratiu, Geometric mechanics, Lagrangian reduction, and nonholonomic systems, In: Mathematics Unlimitted-2001 and beyond, Springer New York, 2001.

[10] J-P. Ortega and T.S. Ratiu, Momentum maps and Hamiltonian reduction, Birkhöuser, Boston, 2004.

[11] J.E. Marsden, G. Misolek, M. Perlmutter, and T.S. Ratiu, Diff. Geom. and its Appl., 9, 173-212 (1998),

[12] J.E. Marsden, G. Misolek, M. Perlmutter, J-P. Ortega, and T.S. Ratiu, Hamiltonian Reduction by Stages, Lec. Notes in Math., vol. 1913, Springer-Verlag, Berlin, 2007.

[13] A. Guichardet, Ann. Inst. H. Poincaré, 40, 329-342 (1984).

[14] T. Iwai, J. Math. Phys., 28, 1315-1326 (1987).

[15] M. Kummer, Indiana Univ. Math. J., 30, 281-291 (1981).

[16] R. Salmon, Ann. Rev. Fluid Mech., 20, 225-256 (1988).

[17] D.D. Holm, J.E. Marsden, and T.S. Ratiu, Adv. Math., 137, 1-81 (1998).

[18] J.E. Marsden and M. Perlmutter, http://www.cds.caltech.edu/ marsden/bib/2000.html (200).

[19] D.D. Holm, arXiv:0708.1585v1 (2007).

[20] J.E. Marsden and J. Scheurle, Fields Institute Communications, 1, 139-164 (1993).

[21] J.C. Simo, D. Lewis, and J.E. Marsden, Arch. Rat. Mech. Anal., 115, 15-59 (1991).

[22] F. Fassò and D. Lewis, Arch. Rational. Mech. Anal. 158, 259-252 (2001). 
[23] A. Hernández-Garduño, J.K. Lawson, and J.E. Marsden, J. Geom. Phys., 53, 259-274 (2005).

[24] T. Iwai, J. Math. Phys., 43, 2927-2947 (2002).

[25] J.E. Marsden, T. Ratiu, and A. Weinstein, Trans. Amer. Math. Soc., 281, 147-177 (1984).

[26] V. Guillemin and S. Sternberg, Ann. Phys., 127, 220-253 (1980).

[27] G. Rosensteel and D.J. Rowe, Ann. Phys., 96, 1-42 (1976).

[28] G. Rosensteel and E. Ihrig, Ann. Phys., 121, 113-130 (1979).

[29] O. Castanõs, A. Frank, E. Chacón, P. Hess, and M. Moshinsky, J. Math. Phys., 23, 2537-2553 (1982).

[30] K.A. Mitchell and R.G. Littelejohn, J. Phys. A: Math. Gen, 33, 1395-1416 (2000).

[31] T. Iwai, J. Phys. A: Math. Theor. 40, 12161-12184 (2007).

[32] D. Lewis and J.C. Simo, Proc. R. Soc. Lond., A427, 281-319 (1990).

[33] T. Iwai, Ann. Inst. Henri Poincaré, 47, 199-219 (1987).

[34] T. Iwai and H. Yamaoka, J. Phys., A38, 2415-2439 (2005).

[35] T. Iwai and H. Yamaoka, J. Phys., A38, 5709-5730 (2005). 